\title{
Homotopy representations of the unitary groups
}

\author{
WOJCIECH LUBAWSKI \\ KRZYSZTOF ZIEMIAŃSKI
}

Let $G$ be a compact connected Lie group and let $\xi$, $v$ be complex vector bundles over the classifying space $B G$. The problem we consider is whether $\xi$ contains a subbundle which is isomorphic to $v$. The necessary condition is that for every prime $p$, the restriction $\left.\xi\right|_{B N_{p}^{G}}$, where $N_{p}^{G}$ is a maximal $p$-toral subgroup of $G$, contains a subbundle isomorphic to $\left.v\right|_{B N_{p}^{G}}$. We provide a criterion when this condition is sufficient, expressed in terms of $\Lambda^{*}$-functors of Jackowski, McClure \& Oliver, and we prove that this criterion applies for bundles $v$ which are induced by unstable Adams operations, in particular for the universal bundle over $B U(n)$. Our result makes it possible to construct new examples of maps between classifying spaces of unitary groups. While proving the main result, we develop the obstruction theory for lifting maps from homotopy colimits along fibrations, which generalizes the result of Wojtkowiak.

$55 \mathrm{R} 37 ; 55 \mathrm{~S} 35$

\section{Introduction}

Let $G$ be a compact connected Lie group. For every prime $p$, there exists a maximal $p$-toral subgroup $N_{p}^{G} \subseteq G$ which is unique up to conjugation. Its identity component is a maximal torus of $G$, and the group of its components is a $p$-Sylow subgroup of the Weyl group of $G$. The following property is the main subject of this paper.

Definition 1.1 A vector bundle $v$ over a space $X$ has the splitting property with respect to a map $f: A \rightarrow X$ if the following holds for an arbitrary bundle $\xi$ over $X$. If there exists a bundle $\xi_{A}^{\prime}$ such that $f^{*} \xi \simeq f^{*} v \oplus \xi_{A}^{\prime}$, then there exists a bundle $\xi^{\prime}$ such that $\xi \simeq v \oplus \xi^{\prime}$. A complex vector bundle over the classifying space $B G$ of $G$ has the splitting property if it has the splitting property with respect to the map

$$
\coprod_{p \text { prime }} B N_{p}^{G} \rightarrow B G
$$

induced by the inclusions $N_{p}^{G} \subseteq G$. 
The splitting property is especially useful due to a theorem of Notbohm [10], which generalizes a result of Dwyer and Zabrodsky [4]. It implies that isomorphism classes of complex vector bundles over $B N_{p}^{G}$ are in one-to-one correspondence with isomorphism classes of unitary representations of $N_{p}^{G}$. Jackowski and Oliver [12] proved that the trivial bundle over $B G$ has the splitting property. In the present paper we extend their result to a wider class of bundles over $B U(n)$. For an arbitrary integer $k$ prime to $n$ !, Sullivan [13] constructed a map $\Psi^{k}: B U(n) \rightarrow B U(n)$ known as the unstable Adams operation, which when restricted to the diagonal matrices is induced by the $k$-power homomorphism. As was shown later by Jackowski, McClure and Oliver [5; 6], this condition determines such maps up to homotopy. Let $\gamma^{n}$ denote the universal bundle over $B U(n)$. The main result of this paper is the following theorem.

Theorem 1.2 For all $n$ and all $k$ prime to $n$ !, the bundle $\left(\Psi^{k}\right)^{*} \gamma^{n}$ over $B U(n)$ has the splitting property.

In particular, for $k=1$ this implies that the universal bundle has the splitting property.

Vector bundles over the classifying space of the group $G$ can be interpreted as maps $B G \rightarrow B U(d)$ which will be called homotopy representations of $G$. The natural source of homotopy representations are linear representations of $G$, ie homomorphisms $G \rightarrow U(n)$. The vector bundles corresponding to maps induced by linear representations are the bundles associated with the universal $G$-bundle $E G \rightarrow B G$. Thus the splitting property of vector bundles can be easily formulated in terms of homotopy theory.

To every homotopy representation $f: B G \rightarrow B U(d)$ we associate a virtual character $\chi_{f} \in R(G)$. Let $T \subseteq G$ be a maximal torus of $G$. By the Dwyer-Zabrodsky-Notbohm theorem [10], the restriction $\left.f\right|_{B T}: B T \rightarrow B U(d)$ is defined by a linear representation $\rho_{f}: T \rightarrow U(d)$ which is unique up to isomorphism, and its character $\chi_{\rho_{f}}$ is invariant under the Weyl group action on the representation ring $R(T)$. Thus via the classical isomorphism $R(G) \simeq R(T)^{W}$ we can consider it as a virtual character $\chi_{f} \in R(G)$. The virtual characters coming from homotopy representations we will call homotopy characters. A natural question arises: when a virtual character $\alpha \in R(G)$ is a homotopy character? The Dwyer-Zabrodsky-Notbohm theorem provides the following constraint: for every prime $p$ and every $p$-toral subgroup $P \subseteq G$, the restriction of $\alpha$ to $P$ is the character of a linear representation. Such characters of the group $G$ will be called $\mathcal{P}$-characters of $G$. Since $N_{p}$ is a maximal $p$-toral subgroup of $G$, a virtual character $\mu \in R(G)$ is a $\mathcal{P}$-character if for every prime the restriction of $\mu$ to $N_{p}$ is the character of a linear representation. Now we can formulate the splitting property for homotopy representations of compact connected Lie groups. 
Definition 1.3 A homotopy character $\mu \in R(G)$ of a compact connected Lie group $G$ has the splitting property if every $\mathcal{P}$-character $v$ of $G$ such that $\mu+v$ is a homotopy character is also a homotopy character.

Hence, Theorem 1.2 can be reformulated as follows.

Theorem 1.4 For all $n$ and all $k$ prime to $n$ !, the character $\psi^{k}$ of the unstable Adams representation $\Psi^{k}: B U(n) \rightarrow B U(n)$ has the splitting property.

The description of homotopy classes of maps between classifying spaces of compact Lie groups is a classical topic of homotopy theory. It attracted attention of many researchers through decades, but still remains open. Our Theorem 1.2 and [8, Proposition 1.13], which implies that the character of the trivial representation has the splitting property, lead to construction of maps $B U(n) \rightarrow B U(d)$ which cannot be produced by compositions of sums and tensor products of the unstable Adams operations and maps induced by homomorphisms [9]. The crucial observation is that characters of some irreducible representations of $U(n)$ can be written as a nontrivial sum of $\mathcal{P}$-characters $\mu+v$, where $v$ is the character of either the trivial or the identity representation. Then $\mu$ is a homotopy character. This construction allows us to classify homotopy representations of $U(n)$ with dimension bounded by some constant depending on $n$ [9]. Every $\mathcal{P}$-character can be written as the difference of characters of representations. Thus, if characters of all representations had the splitting property, then all $\mathcal{P}$-characters would be homotopy characters.

\section{Criterion for splitting $\mathcal{P}$-characters}

Theorem 1.4 is a consequence of a more general criterion for splitting of $\mathcal{P}$-characters. Before formulating it we need to introduce some definitions. Recall from Jackowski, McClure and Oliver [5; 6] that a $p$-toral group $P \subseteq G$ is $p$-stubborn if $N_{G}(P) / P$ is a finite group and contains no nontrivial normal $p$-subgroups. Let $\mathbb{Z}_{p}^{\wedge}$ be the ring of $p$-adic integers, and, for a finite group $\Gamma$ and a $\mathbb{Z}_{p}^{\wedge}[\Gamma]$-module $M$, let $\Lambda^{i}(\Gamma ; M)$ be the $\Lambda^{*}$-functors introduced by Jackowski, McClure and Oliver [6, Definition 5.3]; see also (2.12).

For a compact Lie group $P$, let $\operatorname{IrRep}(P)$ be the set of isomorphisms classes of irreducible complex representations of $P$. For a representation $\alpha$ of $P$ and $\rho \in \operatorname{IrrRep}(P)$, let $c_{\alpha}^{\rho}$ be the multiplicity of $\rho$ in $\alpha$, ie the number of summands isomorphic to $\rho$ in an arbitrary decomposition of $\alpha$ into a sum of irreducible subrepresentations. Furthermore, let $\operatorname{IrRep}(P, \alpha)=\left\{\rho \in \operatorname{IrRRep}(P): c_{\alpha}^{\rho}>0\right\}$. Any (left) action $N \rightarrow \operatorname{Out}(P)$ of a finite group $N$ on $P$ by outer automorphisms induces a right action on $\operatorname{IrRep}(P)$; furthermore, if $\alpha$ is $N$-invariant, then $\operatorname{IrRep}(P, \alpha)$ is an $N$-subset of $\operatorname{IrrRep}(P)$. 
For a $\mathcal{P}$-character $\xi$ of $G$ and a $p$-stubborn subgroup $P \subseteq G$, let $\xi P$ denote a representation with character $\operatorname{res}_{P}^{G} \xi$.

Theorem 1.5 Let $G$ be a compact connected Lie group and let $\mu, v$ be $\mathcal{P}$-characters of $G$ such that $\mu+v$ is a homotopy character. Assume that

$$
\Lambda^{i}\left(N_{G}(P) / P ; \mathbb{Z}_{p}^{\wedge}[X]\right)=0
$$

for every prime $p$, every $p$-stubborn subgroup $P \subseteq G$, every $N_{G}(P) / P$-orbit $X \subseteq$ $\operatorname{IrRep}\left(P, \mu_{P}\right) \cap \operatorname{IrrRep}\left(P, v_{P}\right)$ and every $i \geq 3$. Then both $\mu$ and $v$ are homotopy characters.

The reason this criterion applies when $v=\psi^{k}$ is the character of the unstable Adams operation is the following: for every $p$-stubborn subgroup $P \subseteq U(n)$, there exists a presentation

$$
P \simeq P_{1}^{b_{1}} \times \cdots \times P_{j}^{b_{j}} \subseteq U\left(n_{1}\right)^{b_{1}} \times \cdots \times U\left(n_{j}\right)^{b_{j}} \subseteq U(n)
$$

such that $P_{i} \subseteq U\left(n_{i}\right)$ are pairwise nonisomorphic irreducible $p$-stubborn subgroups. The irreducibility of $P_{i}$ means that the representation $\iota_{P_{i}}$ is irreducible, where $\iota$ denotes the character of the identity. It turns out that also the representations $\psi_{P_{i}}^{k}$ are irreducible. As a consequence, the representation $\psi_{P}^{k}$ admits a decomposition

$$
V=\left(V_{1,1} \oplus \cdots \oplus V_{1, b_{1}}\right) \oplus \cdots \oplus\left(V_{j, 1} \oplus \cdots \oplus V_{j, b_{j}}\right),
$$

where each $V_{i, r}$ is an irreducible representation of the $r^{\text {th }}$ factor of type $P_{i}$, on which the other factors act trivially. The summands in parentheses correspond to the orbits of the $N_{U(n)}(P) / P$-action on $\operatorname{IrrRep}\left(P, \psi_{P}^{k}\right)$. This presentation makes it possible to calculate the groups $\Lambda^{*}$ of Theorem 1.5.

\section{Lifting maps from homotopy colimits}

An important tool used in the proof of Theorem 1.5 is the obstruction theory for lifting maps from homotopy colimits. Let $\mathcal{C}$ be a small category, $F: \mathcal{C} \rightarrow \mathbf{S p}$ a diagram of spaces and $X$ a space. Consider a collection of maps $\left\{f_{c}: F(c) \rightarrow X\right\}_{c \in \mathrm{Ob}(\mathcal{C})}$ that is homotopy compatible, ie for every morphism $c \stackrel{\alpha}{\longrightarrow} c^{\prime}$, the maps $f_{c^{\prime}} \circ F(\alpha)$ and $f_{c}$ are homotopic. By Wojtkowiak [14], the existence of an extension of $\bigsqcup f_{c}$ : $F(c) \rightarrow X$ to a map $f: \operatorname{hocolim}_{\mathcal{C}} F \rightarrow X$ depends on the vanishing of certain obstructions lying in the groups

$$
H^{i+1}\left(\mathcal{C} ; \pi_{i}\left(\operatorname{map}(F(-), X)_{f_{(-)}}\right)\right) .
$$

In this paper we consider a more general problem. Let $p: Y \rightarrow Z$ be a fibration. Fix a map $f: \operatorname{hocolim}_{\mathcal{C}} F \rightarrow Z$ and its partial lifting $\bigsqcup g_{c}: \bigsqcup F(c) \rightarrow Y$ (see (3.1)) 
such that the collection $\left\{g_{c}\right\}_{c \in \mathrm{Ob}(\mathcal{C})}$ is homotopy compatible. We prove in Theorem 3.4 that under certain assumptions, this lifting can be extended to $\operatorname{hocolim}_{\mathcal{C}} F$ if the groups

$$
H^{i+1}\left(\mathcal{C} ; \pi_{i}(\operatorname{Fib}(-))\right)
$$

vanish for $i>0$, where Fib: $\mathcal{C}^{\mathrm{op}} \rightarrow \mathbf{H S p}$ is a certain functor such that $\operatorname{Fib}(c)$ is a fiber of the fibration

$$
\operatorname{map}(F(c), Y)_{g_{c}} \rightarrow \operatorname{map}(F(c), Z)_{\left.f\right|_{F(c)}} .
$$

Organization of the paper In Section 2 we provide definitions and recall theorems used throughout the rest of the paper. In Section 3 we develop the obstruction theory for lifting maps from homotopy colimits. Section 4 contains the proofs of Theorem 1.4 and Theorem 1.5, although the proofs of some propositions are postponed to later sections. We prove Proposition 4.12 in Section 5, Proposition 4.13 in Section 6, and Sections 7 and 8 contain the proof of Theorem 4.9.

Acknowledgements The authors would like to thank the referee for putting in so much work reading this paper and for many valuable suggestions and comments, especially for pointing out a simplification of the proof of Proposition 4.13 and a gap in the proof of Theorem 4.9 in the first version of this paper. We would also like thank Stefan Jackowski for his advice during the writing of this paper.

\section{Preliminaries}

For compact Lie groups $H$ and $L$, let $R(H)$ be the unitary representation ring of $H$, $R^{+}(H) \subseteq R(H)$ the semiring of isomorphism classes of unitary representations of $H$ and let $\operatorname{Rep}(H, L):=\operatorname{Hom}(H, L) / \operatorname{Inn}(L)$ be the set of conjugacy classes of homomorphisms from $H$ to $L$. If $H \subseteq L$, then $C_{L}(H)$ denotes the centralizer of $H$ in $L$. For a space $X$, we denote by $X_{p}^{\wedge}$ the $\mathbb{F}_{p}$-completion in the sense of Bousfield and Kan [3].

\section{Dwyer-Zabrodsky-Notbohm theorem}

An important tool we use in this paper is the following theorem due to Dwyer, Zabrodsky and Notbohm.

Theorem 2.1 (Dwyer-Zabrodsky-Notbohm theorem [10]) Let $P$ be a $p$-toral group and $H$ a compact Lie group.

- The map

$$
\operatorname{Rep}(P, H) \rightarrow[B P, B H], \quad \alpha \mapsto[B \alpha]
$$

is a bijection. 
- For every homomorphism $\alpha: P \rightarrow H$ the map

$$
\operatorname{ad}_{\alpha}: B C_{H}(\alpha(P)) \rightarrow \operatorname{map}(B P, B H)_{B \alpha},
$$

which is adjoint to the map induced by the multiplication homomorphism

$$
C_{H}(\alpha(P)) \times P \rightarrow H, \quad(a, b) \mapsto \alpha(a) b,
$$

is a mod $p$-equivalence, ie it induces an isomorphism in homology with $\mathbb{Z} / p$ as coefficients. In particular, the map

$$
\left(\operatorname{ad}_{\alpha}\right)_{p}^{\wedge}: B C_{H}(\alpha(P))_{p}^{\wedge} \rightarrow\left(\operatorname{map}(B P, B H)_{B \alpha}\right)_{p}^{\wedge} \cong \operatorname{map}\left(B P, B H_{p}^{\wedge}\right)_{B \alpha}
$$

is a homotopy equivalence.

If $H=U(d)$ is a unitary group, then $C_{H}(\rho(P))$ can be described using Schur's lemma. Namely, there is an isomorphism

$$
C_{U(d)}(\alpha(P)) \simeq \prod_{\rho \in \operatorname{IrrRep}(P)} U\left(c_{\alpha}^{\rho}\right) .
$$

\section{$\mathcal{P}$-characters and homotopy characters}

Fix a compact connected Lie group $G$. Let $T \subseteq G$ be its maximal torus, and $W \subseteq \operatorname{Aut}(T)$ its Weyl group.

Definition 2.3 A homotopy representation of $G$ is a map $f: B G \rightarrow B U(d)$. We say that two homotopy representations are isomorphic if they are homotopic as maps. The character $\chi(f) \in R(G) \cong R(T)^{W}$ of a homotopy representation $f$ is the character of the representation $\rho: T \rightarrow U(d)$ such that $\left.B \rho \sim f\right|_{B T}$. By Theorem 2.1, such a character is well-defined.

Definition 2.4 A virtual character $\mu \in R(G)$ is a $\mathcal{P}$-character if for every prime $p$ and every $p$-toral subgroup $P \subseteq G$, its restriction to $P$ is the character of a linear representation, ie $\left.\mu\right|_{P} \in R^{+}(P)$. The set of $\mathcal{P}$-characters of $G$ will be denoted by $R_{\mathcal{P}}(G)$. We will denote by $\mu_{P}: P \rightarrow U(d)$ a homomorphism with the character $\left.\mu\right|_{P}$.

Proposition 2.5 The character of a homotopy representation is a $\mathcal{P}$-character.

Proof If $f: B G \rightarrow B U(d)$ is a homotopy representation and $P \subseteq G$ a $p$-subgroup, then the Dwyer-Zabrodsky-Notbohm theorem implies that the restriction $\left.f\right|_{B P}$ is induced by a unitary representation of $P$. 
Definition 2.6 A virtual character that is the character of a homotopy representation will be called a homotopy character; the semiring of homotopy characters of $G$ will be denoted by $R_{h}(G)$.

Notice that there is a sequence of inclusions

$$
R^{+}(G) \subseteq R_{h}(G) \subseteq R_{\mathcal{P}}(G) \subseteq R(G) \cong R(T)^{W} \subseteq R(T)
$$

\section{p-homotopy characters}

Definition 2.8 Let $p$ be a prime integer. A $p$-homotopy representation of $G$ is a map $f_{p}: B G \rightarrow B U(d)_{p}^{\wedge}$. We say that a $\mathcal{P}$-character $\mu \in R_{\mathcal{P}}(G)$ is the character of $f_{p}$ if the diagram

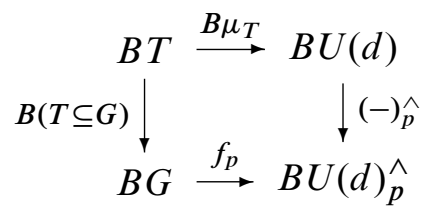

commutes up to homotopy. A $\mathcal{P}$-character that is the character of a $p$-homotopy representation is called a $p$-homotopy character.

Proposition 2.9 A $\mathcal{P}$-character $\mu$ is a homotopy character if and only if it is a $p-$ homotopy character for all primes $p$.

Proof This is an immediate consequence of [7, Proposition 1.2].

\section{The subgroup homotopy decomposition}

Let $p$ be a prime integer.

Definition 2.10 A p-toral subgroup $P \subseteq G$ is $p$-stubborn if $N_{G}(P) / P$ is a discrete group containing no nontrivial normal $p$-subgroups. Let $\mathcal{R}_{p}(G)$ be the category of $G$-orbits with the form $G / P$, for a $p$-stubborn $P \subseteq G$, and $G$-maps.

Theorem 2.11 [5, Theorem 1.4] The map

$$
\varepsilon_{G}^{p}: \operatorname{hocolim}_{G / P \in \mathcal{R}_{p}(G)} E G \times_{G} G / P \rightarrow E G \times_{G} * \cong B G
$$

induced by projections $G / P \rightarrow *$ induces an equivalence on homology with $\mathbb{F}_{p}-$ coefficients. In particular, the map

$$
\left(\varepsilon_{G}^{p}\right)_{p}^{\wedge}:\left(\operatorname{hocolim}_{G / P \in \mathcal{R}_{p}(G)} E G \times_{G} G / P\right)_{p}^{\wedge} \rightarrow B G_{p}^{\wedge}
$$

is a homotopy equivalence. 
Remark If $\mu$ is a $\mathcal{P}$-character of $G$ of dimension $d$, then the family

$$
E G \times_{G} G / P \simeq B P \stackrel{B \mu_{P}}{\longrightarrow} B U(d)
$$

is a homotopy compatible family of maps from the decomposition functor $E G \times_{G}(-)$ to $B U(d)$.

\section{Functors $\Lambda^{*}$}

Let $p$ be a prime. Here we recall methods of calculating higher limits of functors on the category $\mathcal{R}_{p}(G)$ developed in $[5 ; 6]$. For a commutative ring $R$ and a small category $\mathcal{C}$, we define $R[\mathcal{C}]$-modules as contravariant functors from $\mathcal{C}$ into the category of $R$-modules. If $F$ is an $R[\mathcal{C}]$-module, then $H^{i}(\mathcal{C} ; F)$ denotes the $i^{\text {th }}$ right derived functor of the inverse limit of $F$, and will be referred to as the $i^{\text {th }}$ cohomology group of $\mathcal{C}$ with coefficients $M$; this notion coincides with group cohomology if $\mathcal{C}$ is a one-object category with invertible morphisms.

For a finite group $\Gamma$, let $\mathcal{O}_{p}(\Gamma)$ be the category of $\Gamma$-orbits whose isotropy groups are $p$-groups and $\Gamma$-maps. For a $\mathbb{Z}_{p}^{\wedge}[\Gamma]$-module $M$ and $i \geq 0$, define groups

$$
\Lambda^{i}(\Gamma ; M):=H^{i}\left(\mathcal{O}_{p}(\Gamma) ; F_{M}^{\Gamma}\right),
$$

where $F_{M}^{\Gamma}$ is a $\mathbb{Z}_{p}^{\wedge}\left[\mathcal{O}_{p}(\Gamma)\right]$-module defined by

$$
F_{M}^{\Gamma}(\Gamma / P)= \begin{cases}M & \text { for } P=1, \\ 0 & \text { otherwise }\end{cases}
$$

These groups play an important role in calculating cohomology of $\mathbb{Z}_{p}^{\wedge}\left[\mathcal{R}_{p}(G)\right]$-modules. Their crucial property is the following.

Proposition 2.14 Let $G$ be a compact Lie group, $F$ a $\mathbb{Z}_{p}^{\wedge}\left[\mathcal{R}_{p}(G)\right]$-module and $r$ an integer. Assume that $\Lambda^{i}\left(N_{G}(P) / P ; F(G / P)\right)=0$ for all $i \geq r$ and all $G / P \in \mathcal{R}_{p}(G)$. Then $H^{i}\left(\mathcal{R}_{p}(G) ; F\right)=0$ for $i \geq r$.

Proof This follows from [5, Corollary 1.8] and [7, Theorem 1.10(ii)].

Let us recall several properties of functors $\Lambda^{*}$ proven in $[5 ; 6]$.

Proposition 2.15 Let $\Gamma$ be a finite group and let $M$ be a $\mathbb{Z}_{p}^{\wedge}[\Gamma]-$ module.

(1) If $p$ divides the order of $\Gamma$, then $\Lambda^{0}(\Gamma ; M)=0$. Otherwise $\Lambda^{0}(\Gamma ; M)=M^{\Gamma}$.

(2) Let $K=\operatorname{ker}(\Gamma \rightarrow \operatorname{Aut}(M))$. If $p$ divides the order of $K$, then $\Lambda^{*}(\Gamma ; M)=0$; otherwise $\Lambda^{*}(\Gamma ; M)=\Lambda^{*}(\Gamma / K ; M)$.

Proof This follows from [6, Proposition 6.1]. 
In the following proposition all tensor products are taken with respect to the ring $\mathbb{Z}_{p}^{\wedge}$.

Proposition 2.16 Let $\Gamma$ be a finite group, $M$ a finitely generated $\mathbb{Z}_{p}^{\wedge}$-module, $N$ a $\mathbb{Z}_{p}^{\wedge}[\Gamma]$-module that is free and finitely generated as $\mathbb{Z}_{p}^{\wedge}$-module, and $r \geq 0$ an integer. Assume that $\Lambda^{i}(\Gamma ; N)=0$ for every $i \geq r$. Then $\Lambda^{i}(\Gamma ; M \otimes N)=0$ for $i \geq r$.

Proof By [6, Proposition 6.1(v)] there is an exact sequence

$$
\begin{aligned}
0 \rightarrow \bigoplus_{k+l=i+1} \operatorname{Tor}\left(\Lambda^{k}(1 ; M), \Lambda^{l}(\Gamma ; N)\right) \rightarrow \Lambda^{i}(\Gamma ; M \otimes N) & \\
& \rightarrow \bigoplus_{k+l=i} \Lambda^{l}(1 ; M) \otimes \Lambda^{k}(\Gamma ; N) \rightarrow 0,
\end{aligned}
$$

and by [6, Proposition 6.1(i)] it reduces to

$$
0 \rightarrow \operatorname{Tor}\left(M, \Lambda^{i+1}(\Gamma ; N)\right) \rightarrow \Lambda^{i}(\Gamma ; M \otimes N) \rightarrow M \otimes \Lambda^{i}(\Gamma ; N) \rightarrow 0 .
$$

By assumption $\Lambda^{i}(\Gamma ; N)=\Lambda^{i+1}(\Gamma ; N)=0$. The conclusion follows.

\section{Obstruction theory}

In this section we develop the obstruction theory for lifting maps from homotopy colimits. This is an extension of results obtained by Wojtkowiak [14]. Let $\mathcal{C}$ be a small category, $F: \mathcal{C} \rightarrow \mathbf{S p}$ a diagram of spaces and $p: Y \rightarrow Z$ a fibration. Fix a map $f: \operatorname{hocolim}_{\mathcal{C}} F \rightarrow Z$ and a family of maps $g_{c}: F(c) \rightarrow Y, c \in \mathrm{Ob}(\mathcal{C})$ which represents a co-cone in the homotopy category, ie an element

$$
\left\{\left[g_{c}\right]\right\}_{c \in \mathrm{Ob}(\mathcal{C})} \in \lim _{c \in \mathcal{C}}[F(c), Y] .
$$

Assume that the diagram of solid arrows

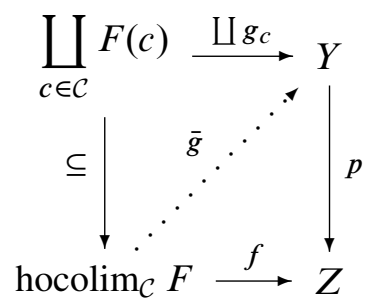

strictly commutes. For every $c \in \operatorname{Ob}(\mathcal{C})$ denote $f_{c}:=\left.f\right|_{F(c)}$ and

$$
\operatorname{Fib}(c):=\left(p_{c}\right)^{-1}\left(f_{c}\right),
$$

where

$$
p_{c}:=\operatorname{map}(F(c), p): \operatorname{map}(F(c), Y)_{g_{c}} \stackrel{p_{*}}{\longrightarrow} \operatorname{map}(F(c), Z)_{f_{c}} .
$$


The map $p_{c}$ is a fibration with fiber $\operatorname{Fib}(c)$. We will make two more assumptions: for every $c \in \mathrm{Ob}(\mathcal{C})$,

- the connected component of the mapping space $\operatorname{map}(F(c), Z)_{f_{c}}$ is simply connected, and

- $\operatorname{Fib}(c)$ is a simple space ie its fundamental group acts trivially on all homotopy groups.

For every morphism $\alpha: c \rightarrow c^{\prime}$ in $\mathcal{C}$, let us choose a map

$$
\operatorname{Fib}(\alpha): \operatorname{Fib}\left(c^{\prime}\right)=p_{c^{\prime}}^{-1}\left(f_{c^{\prime}}\right) \stackrel{F(\alpha)^{*}}{\longrightarrow} p_{c}^{-1}\left(f_{c^{\prime}} \circ F(\alpha)\right) \stackrel{\simeq}{\longrightarrow} p_{c}^{-1}\left(f_{c}\right)=\operatorname{Fib}(c) .
$$

The first assumption guarantees that the homotopy class of $\operatorname{Fib}(\alpha)$ does not depend on the choice of a homotopy equivalence between fibers of $p_{c}$. Thus, Fib is a contravariant functor from $\mathcal{C}$ to the homotopy category HSp. By the second assumption, for every $c \in \operatorname{Ob}(\mathcal{C})$, the homotopy groups of $\operatorname{Fib}(c)$ for different choices of basepoints are naturally isomorphic. Therefore, the composition $\pi_{n} \circ \mathrm{Fib}$ is a contravariant functor from $\mathcal{C}$ into the category of abelian groups $\mathbf{A b}$. The following theorem will be proven at the end of the section.

Theorem 3.4 If $H^{i+1}\left(\mathcal{C} ; \pi_{i}(\mathrm{Fib})\right)=0$ for all $i>0$, then there exists a lifting extension $\bar{g}$ : hocolim $_{\mathcal{C}} F \rightarrow Y$ which makes the diagram (3.1) commutative.

This theorem reduces to the result of [14] if $Z$ is a one-point space.

\section{Elementary lifting extension problem}

To establish notation, we recall some elementary results of classical obstruction theory. Fix a fibration $q: E \rightarrow B$ such that its fiber $F=q^{-1}\left(b_{0}\right)$ is simple, and assume that $B$ is simply connected. Consider the following lifting extension problem:

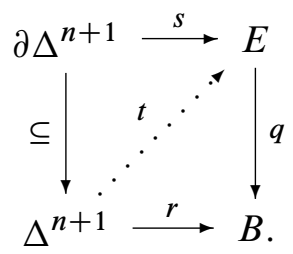

Let

$$
\mathcal{L}(s, r):=\left\{t \in \operatorname{map}\left(\Delta^{n+1}, E\right):\left.t\right|_{\partial \Delta^{n+1}}=s \text { and } q \circ t=r\right\}
$$

be the space of lifting extensions. 
The map $s$ factors through the total space $r^{*} E$ of the pull-back fibration over $\Delta^{n+1}$. Let

$$
\mathfrak{o}_{n}(s, r) \in\left[\partial \Delta^{n+1}, r^{*} E\right] \cong \pi_{n}\left(r^{*} E\right) \simeq \pi_{n}(F)
$$

be the homotopy class corresponding to this factorization; we will call it the obstruction class. This definition depends neither on the choice of basepoints of the homotopy groups (since $F$ is simple), nor on the choice of homotopy equivalence $r^{*} E \simeq F$ (since $B$ is simply connected). For $t, t^{\prime} \in \mathcal{L}(s, r)$, define the difference class

$$
\mathfrak{d}_{n+1}\left(t, t^{\prime}\right) \in\left[\Delta^{n+1} \cup_{\partial \Delta^{n+1}} \Delta^{n+1}, r^{*} E\right] \simeq \pi_{n+1}(F)
$$

as the obstruction class of the lifting extension problem

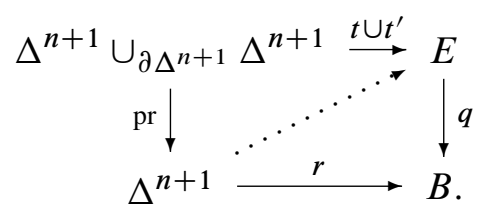

Let $d_{i}: \Delta^{k} \rightarrow \Delta^{k+1}$ be the inclusion onto the $i^{\text {th }}$ face of the simplex, and let $\Delta_{(l)}^{k}$ be the $l^{\text {th }}$ skeleton of $\Delta^{k}$. Let us state some elementary properties of the concepts defined above.

Proposition 3.10 Fix a map $r: \Delta^{n+1} \rightarrow B$.

(1) If $s: \partial \Delta^{n} \rightarrow E$ is a lifting of $\left.r\right|_{\partial \Delta^{n}}$ along $q$, then $\mathcal{L}(s, r) \neq \varnothing$ if and only if $\mathfrak{o}_{n}(s, r)=0$.

(2) Let $s, s^{\prime}: \partial \Delta^{n+1} \rightarrow E$ be liftings of $\left.r\right|_{\partial \Delta^{n+1}}$ along $q$. Assume that $\left.s\right|_{\Delta_{(n-1)}^{n+1}}=$ $\left.s^{\prime}\right|_{\Delta_{(n-1)}^{n+1}}$. Then

$$
\mathfrak{o}_{n}\left(s^{\prime}, r\right)=\mathfrak{o}_{n}(s, r)+\sum_{i=0}^{n+1}(-1)^{i} \mathfrak{d}_{n}\left(s^{\prime} \circ d_{i}, s \circ d_{i}\right) .
$$

(3) We have $\sum_{i=0}^{n+2}(-1)^{i} \mathfrak{o}_{n}\left(s \circ d_{i}, r \circ d_{i}\right)=0$ for any commutative diagram

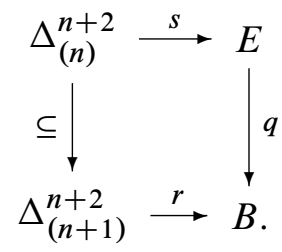

(4) If $s: \partial \Delta^{n} \rightarrow E$ is a lifting of $\left.r\right|_{\partial \Delta^{n}}$ along $q$, then, for every $t \in \mathcal{L}(s, r)$ and every $u \in \pi_{n+1}(F)$, there exists $t^{\prime} \in \mathcal{L}(s, r)$ such that $\mathfrak{d}_{n-1}\left(t^{\prime}, t\right)=u$. 
Proposition 3.11 (functoriality of obstruction and difference classes) Consider a commutative diagram

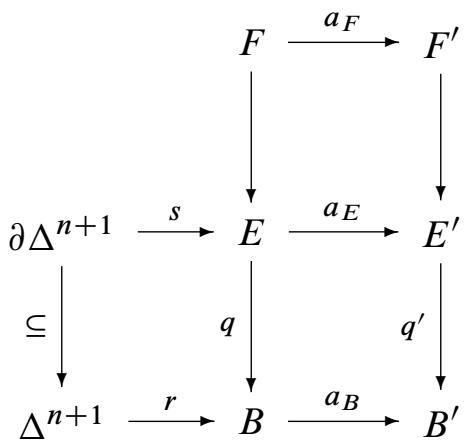

where $q^{\prime}: E^{\prime} \rightarrow B^{\prime}$ is a fibration with a fiber $F^{\prime}$. Assume that $B^{\prime}$ is simply connected and $F^{\prime}$ is simple. Then

(1) $\mathfrak{o}_{n}\left(a_{E} \circ s, a_{B} \circ r\right)=\left(a_{F}\right)_{*}\left(\mathfrak{o}_{n}(s, r)\right)$,

(2) for $t, t^{\prime} \in \mathcal{L}(s, r)$,

$$
\mathfrak{d}_{n+1}\left(a_{E} \circ t, a_{E} \circ t^{\prime}\right)=\left(a_{F}\right)_{*} \mathfrak{d}_{n+1}\left(t, t^{\prime}\right) .
$$

\section{Cochain complex}

We return to considering the lifting extension problem (3.1). Here we define a cochain complex which can be used to calculating cohomology groups $H^{j}\left(\mathcal{C} ; \pi_{i}(\mathrm{Fib})\right)$. Also the obstruction classes and the difference classes, which are analogues of (3.7) and (3.8), will be defined as its cochains.

Let $N(\mathcal{C})$ denote the nerve of $\mathcal{C}$ and let $N(\mathcal{C})_{i}$ be the set of $i$-simplices of $N(\mathcal{C})$. For $i, j>0$ define groups

$$
C_{i}^{j}=\prod_{\sigma \in N(\mathcal{C})_{j}} \pi_{i}(\operatorname{Fib}(\sigma(0)))
$$

and differentials $\delta_{i}^{j}: C_{i}^{j} \rightarrow C_{i}^{j+1}$ by

$$
\delta_{i}^{j}(u)(\sigma)=\operatorname{Fib}(\sigma(0 \rightarrow 1))^{*}\left(u\left(d_{0} \sigma\right)\right)+\sum_{k=1}^{j+1}(-1)^{k} u\left(d_{k}(\sigma)\right)
$$

for $u \in C_{i}^{j}, \sigma \in N(\mathcal{C})_{j+1}$. Let $Z_{i}^{j}, B_{i}^{j}, H_{i}^{j}$ denote respectively the cocycles, the coboundaries and the cohomology of the cochain complex $\left(C_{i}^{*}, \delta_{i}^{*}\right)$. According to [11, Lemma 2], $H^{j}\left(\mathcal{C} ; \pi_{i}(\mathrm{Fib})\right)=H_{i}^{j}$ for all $i, j$. 


\section{Adjoint maps}

Denote for short $X=\operatorname{hocolim}_{\mathcal{C}} F$, and let $X_{k}=\operatorname{hocolim}_{\mathcal{C}}^{(k)} F$ be the $k$-skeleton of the homotopy colimit of $F$, ie the inverse image of the $k$-skeleton of the nerve of $\mathcal{C}$. For a space $W$, a map $a: X_{k} \rightarrow W$ and a simplex $\sigma \in N(\mathcal{C})_{n}$, let

$$
\operatorname{Ad}_{\sigma}^{a}: \Delta_{(k)}^{n} \rightarrow \operatorname{map}(F(\sigma(0), W))
$$

be the adjoint map to the composition

$$
\Delta_{(k)}^{n} \times F(\sigma(0)) \stackrel{\sigma}{\longrightarrow} \operatorname{hocolim}_{\mathcal{C}}^{(k)} F=X_{k} \stackrel{a}{\longrightarrow} W .
$$

Note that

$$
\operatorname{Ad}_{\sigma}^{a} \circ d_{i}= \begin{cases}\operatorname{Ad}_{d_{i} \sigma}^{a} & \text { for } i>0, \\ F(\sigma(0 \rightarrow 1)) \circ \operatorname{Ad}_{d_{0} \sigma}^{a} & \text { for } i=0,\end{cases}
$$

and that maps $a: X_{k} \rightarrow W$ are in one-to-one correspondence with families of maps $\left\{\operatorname{Ad}_{\sigma}^{a}\right\}_{\sigma \in N(\mathcal{C})}$ satisfying these relations.

\section{Lifting extension spaces}

For $n \geq 0$, let $\mathcal{E}_{0}^{n}$ be the space of maps $g: X_{n} \rightarrow Y$ such that the diagram

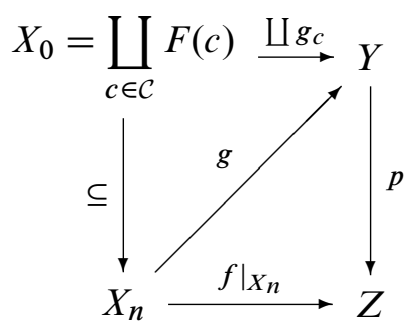

commutes. Denote by $\mathcal{E}_{0}^{\infty}$ the inverse $\operatorname{limit}_{\lim } \mathcal{E}_{0}^{n}$; note that this is the set of solutions of the lifting extension problem (3.1). For $g \in \mathcal{E}_{0}^{n}$ and $k \leq n \leq m$, let $\mathcal{E}_{k}^{m}(g)$ be the space of maps $h: X_{m} \rightarrow Y$ such that the diagram

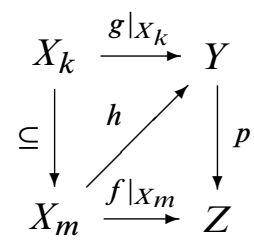

commutes. 


\section{Obstruction cochains and difference cochains}

For $g \in \mathcal{E}_{0}^{n}$, define the obstruction cochain $\mathfrak{O}_{n}(g) \in C_{n}^{n+1}$ by

$$
\mathfrak{O}_{n}(g)(\sigma):=\mathfrak{o}_{n}\left(\operatorname{Ad}_{\sigma}^{g}, \operatorname{Ad}_{\sigma}^{f}\right) \in \pi_{n}(\operatorname{Fib}(\sigma(0))),
$$

where $\mathfrak{o}_{n}\left(\operatorname{Ad}_{\sigma}^{g}, \operatorname{Ad}_{\sigma}^{f}\right)$ is the obstruction of the lifting problem

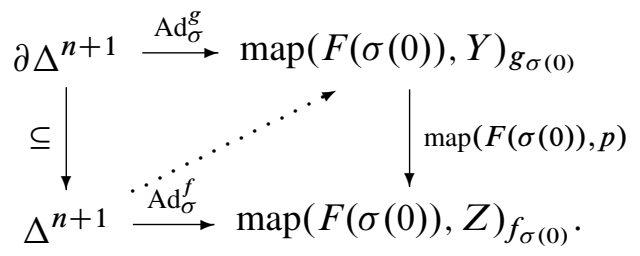

For $h, h^{\prime} \in \mathcal{E}_{n}^{n+1}(g)$, define the difference cochain $\mathfrak{D}_{n+1}\left(h^{\prime}, h\right) \in C_{n+1}^{n+1}$ by

$$
\mathfrak{D}_{n+1}\left(h^{\prime}, h\right)(\sigma)=\mathfrak{d}_{n+1}\left(\operatorname{Ad}_{\sigma}^{h^{\prime}}, \operatorname{Ad}_{\sigma}^{h}\right) \in \pi_{n+1}(\operatorname{Fib}(\sigma(0))) .
$$

Proposition 3.21 Fix $g \in \mathcal{E}_{0}^{n}, n>0$.

(1) $\mathcal{E}_{n}^{n+1}(g) \neq \varnothing$ if and only if $\mathfrak{O}_{n}(g)=0$.

(2) $\mathfrak{O}_{n}(g) \in Z_{n}^{n+1}$.

(3) $\mathfrak{O}_{n}\left(g^{\prime}\right)=\mathfrak{O}_{n}(g)+\delta_{n}^{n}\left(\mathfrak{D}_{n}\left(g^{\prime}, g\right)\right)$ for any $g^{\prime} \in \mathcal{E}_{n-1}^{n}(g)$.

(4) For every $u \in C_{n+1}^{n+1}$ and every $h \in \mathcal{E}_{n}^{n+1}(g)$, there exists a map $h^{\prime} \in \mathcal{E}_{n}^{n+1}(g)$ such that $\mathfrak{D}_{n+1}\left(h^{\prime}, h\right)=u$.

Proof The existence of $h \in \mathcal{E}_{n}^{n+1}(g)$ is equivalent to the existence of a family of maps $\left\{\operatorname{Ad}_{\sigma}^{h}\right\} \in \mathcal{L}\left(\operatorname{Ad}_{\sigma}^{g}, \operatorname{Ad}_{\sigma}^{f}\right)$ for $\sigma \in N(\mathcal{C})_{n+1}$. Then (1) is a straightforward consequence of Proposition 3.10(1). For similar reasons, (4) is a consequence of Proposition 3.10(4). To prove (2), we check that for $\sigma \in N(\mathcal{C})_{n+2}$ we have

$$
\begin{aligned}
\delta_{n}^{n+1}\left(\mathfrak{O}_{n}(g)\right)(\sigma)= & \operatorname{Fib}(\sigma(0 \rightarrow 1))^{*}\left(\mathfrak{O}_{n}(g)\left(d_{0} \sigma\right)\right)+\sum_{i=1}^{n+2}(-1)^{i} \mathfrak{O}_{n}(g)\left(d_{i} \sigma\right) \\
= & \operatorname{Fib}(\sigma(0 \rightarrow 1))^{*} \mathfrak{o}_{n}\left(\operatorname{Ad}_{d_{0} \sigma}^{g}, \operatorname{Ad}_{d_{0} \sigma}^{f}\right)+\sum_{i=1}^{n+2}(-1)^{i} \mathfrak{o}_{n}\left(\operatorname{Ad}_{d_{i} \sigma}^{g}, \operatorname{Ad}_{d_{i} \sigma}^{f}\right) \\
= & \mathfrak{o}_{n}\left(\operatorname{Ad}_{d_{0} \sigma}^{g} \circ F^{*}(\sigma(0 \rightarrow 1)), \operatorname{Ad}_{d_{0} \sigma}^{f} \circ F^{*}(\sigma(0 \rightarrow 1))\right) \\
& +\sum_{i=1}^{n+2}(-1)^{i} \mathfrak{o}_{n}\left(\operatorname{Ad}_{d_{i} \sigma}^{g}, \operatorname{Ad}_{d_{i} \sigma}^{f}\right) \\
= & \sum_{i=0}^{n+2}(-1)^{i} \mathfrak{o}_{n}\left(\operatorname{Ad}_{\sigma}^{g} \circ d_{i}, \operatorname{Ad}_{\sigma}^{f} \circ d_{i}\right)=0
\end{aligned}
$$


where the third equality follows from Proposition 3.10(2), the fourth from (3.15), and the last from Proposition 3.10(3).

To prove (3), let $\sigma \in N(\mathcal{C})_{n+1}$. As a consequence of (3.15), for $i>0$ we have

$$
\mathfrak{o}_{n}\left(\operatorname{Ad}_{\sigma}^{g^{\prime}} \circ d_{i}, \operatorname{Ad}_{g}^{\sigma} \circ d_{i}\right)=\mathfrak{o}_{n}\left(\operatorname{Ad}_{d_{i} \sigma}^{g^{\prime}}, \operatorname{Ad}_{d_{i} \sigma}^{g}\right)=\mathfrak{D}_{n}\left(g^{\prime}, g\right)\left(d_{i} \sigma\right)
$$

and

$$
\begin{aligned}
\mathfrak{d}_{n}\left(\mathrm{Ad}_{\sigma}^{g^{\prime}} \circ d_{0}, \operatorname{Ad}_{\sigma}^{g} \circ d_{0}\right) & =\mathfrak{d}_{n}\left(F(\sigma(0 \rightarrow 1)) \circ \operatorname{Ad}_{d_{0} \sigma}^{g^{\prime}}, F(\sigma(0 \rightarrow 1)) \circ \operatorname{Ad}_{d_{0} \sigma}^{g}\right) \\
& =\operatorname{Fib}(\sigma(0,1))_{*}\left(\mathfrak{d}_{n}\left(\operatorname{Ad}_{d_{0} \sigma}^{g^{\prime}}, \operatorname{Ad}_{d_{0} \sigma}^{g}\right)\right) \\
& =\operatorname{Fib}(\sigma(0,1))_{*}\left(\mathfrak{D}_{n}\left(g^{\prime}, g\right)\left(d_{0} \sigma\right)\right),
\end{aligned}
$$

where the second equality follows from Proposition 3.11(2). Finally, with the second equality following from Proposition 3.10(2),

$$
\begin{aligned}
\mathfrak{O}_{n}\left(g^{\prime}\right)(\sigma) & =\mathfrak{o}_{n}\left(\operatorname{Ad}_{\sigma}^{g^{\prime}}, \operatorname{Ad}_{\sigma}^{f}\right)=\mathfrak{o}_{n}\left(\operatorname{Ad}_{\sigma}^{g}, \operatorname{Ad}_{\sigma}^{f}\right)+\sum_{i=0}^{n+1}(-1)^{i} \mathfrak{d}_{n}\left(\operatorname{Ad}_{\sigma}^{g^{\prime}} \circ d_{i}, \operatorname{Ad}_{\sigma}^{g} \circ d_{i}\right) \\
& =\mathfrak{O}_{n}(g)(\sigma)+\operatorname{Fib}(\sigma(0 \rightarrow 1))_{*}\left(\mathfrak{D}_{n}\left(g^{\prime}, g\right)\left(d_{0} \sigma\right)\right)+\sum_{i=1}^{n+1}(-1)^{i} \mathfrak{D}_{n}\left(g^{\prime}, g\right)\left(d_{i} \sigma\right) \\
& =\mathfrak{O}_{n}(g)(\sigma)+\delta_{n}^{n}\left(\mathfrak{D}_{n}\left(g^{\prime}, g\right)\right) .
\end{aligned}
$$

This proves (3).

Lemma 3.22 Fix $n>0$, and assume that $H^{n+1}\left(\mathcal{C} ; \pi_{n}(\right.$ Fib $\left.)\right)=0$. For every $g \in \mathcal{E}_{0}^{n}$, we have $\mathcal{E}_{n-1}^{n+1}(g) \neq \varnothing$.

Proof By Proposition 3.21(2), $\mathfrak{O}_{n}(g) \in Z_{n}^{n+1}$. Since $H_{n}^{n+1}=0$, there exists $u \in C_{n}^{n}$ such that $\delta_{n}^{n}(u)=\mathfrak{O}_{n}(g)$. Let $g^{\prime} \in \mathcal{E}_{n-1}^{n}(g)$ be a map such that $\mathfrak{D}_{n}\left(g^{\prime}, g\right)=-u$, which exists by Proposition 3.21(4). By Proposition 3.21(3) we have

$$
\mathfrak{O}_{n}\left(g^{\prime}\right)=\mathfrak{O}_{n}(g)+\delta_{n}^{n}\left(\mathfrak{D}_{n}\left(g^{\prime}, g\right)\right)=\mathfrak{O}_{n}(g)+\delta_{n}^{n}(-u)=0 .
$$

The conclusion follows from Proposition 3.21(1).

Finally, we are ready to prove the main theorem of this section.

Proof of Theorem 3.4 We need to prove that the space $\mathcal{E}_{0}^{\infty}=\lim \mathcal{E}_{0}^{n}$ is nonempty. Since $\left\{g_{c}\right\}_{c \in \mathrm{Ob}(\mathcal{C})} \in \lim _{\mathcal{C}}[F, Y]$, the map $g_{0}=\coprod g_{c}: X_{0} \rightarrow Y$ extends to a map $X_{1} \rightarrow Y$, which can be adjusted (since $p$ is a fibration) to a map $g_{1} \in \mathcal{E}_{0}^{1}$. Using Lemma 3.22, we inductively construct a sequence of maps $g_{n}, n \geq 2$, such that $g_{n} \in \mathcal{E}_{n-2}^{n}\left(g_{n-1}\right)$. Put $g_{n}^{\prime}:=\left.g_{n+1}\right|_{X_{n}}$. Since $\left.g_{n}^{\prime}\right|_{X_{n-1}}=g_{n-1}^{\prime}$, the sequence $\left\{g_{n}^{\prime}\right\}$ represents an element in $\mathcal{E}_{0}^{\infty}$. 


\section{Guide to the argument}

In this section we prove Theorem 1.5 and then deduce Theorem 1.4. Let $G$ be a compact connected Lie group and $T \subseteq G$ its maximal torus. Let $\mu$ and $\nu$ be $\mathcal{P}-$ characters of $G$ with dimensions $d$ and $d^{\prime}$, respectively, and assume that $\mu+v$ is a homotopy character. To prove Theorem 1.5 we need to show that under certain assumptions, both $\mu$ and $v$ are homotopy characters. By Proposition 2.9 it is sufficient to prove that they are $p$-homotopy characters for all primes $p$. Fix a prime $p$ and a $p$-homotopy representation $f: B G \rightarrow B U\left(d+d^{\prime}\right)_{p}^{\wedge}$ with character $\mu+v$. We will prove a sequence of criteria (Proposition 4.1, Proposition 4.4, Theorem 4.10) which imply that $\mu$ and $v$ are $p$-homotopy characters.

\section{A lifting extension problem}

The first step in the proof of Theorem 1.5 is the following observation.

Proposition 4.1 If there exists a map $g: B G \rightarrow\left(B U(d) \times B U\left(d^{\prime}\right)\right)_{p}^{\wedge}$ such that the diagram

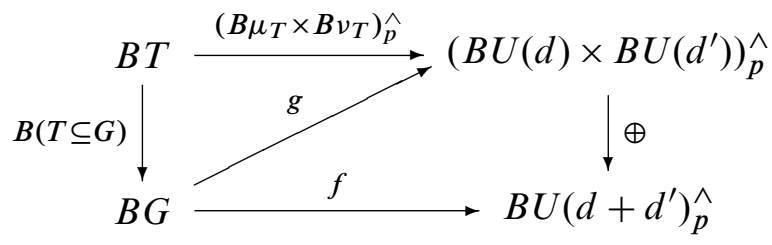

commutes up to homotopy, then $\mu$ and $v$ are $p$-homotopy characters.

Proof The restriction of the composition

$$
f_{1}: B G \stackrel{g}{\longrightarrow}\left(B U(d) \times B U\left(d^{\prime}\right)\right)_{p}^{\wedge} \stackrel{\text { proj }}{\longrightarrow} B U(d)_{p}^{\wedge}
$$

to $B T$ is homotopic to $\left(B \mu_{T}\right)_{p}^{\wedge}$. Therefore, $f_{1}$ is a $p$-homotopy representation with character $\mu$. A similar argument applies to $v$.

\section{A homotopy decomposition}

We will use the homotopy decomposition Theorem 2.11 to find criteria for the existence of a lifting extension in the diagram of Proposition 4.1. Consider the following diagram, where $q$ is induced by the identity map on $E U\left(d+d^{\prime}\right)$, and $\varepsilon_{G}^{p}$ is a mod $p$ homology equivalence from Theorem 2.11: 


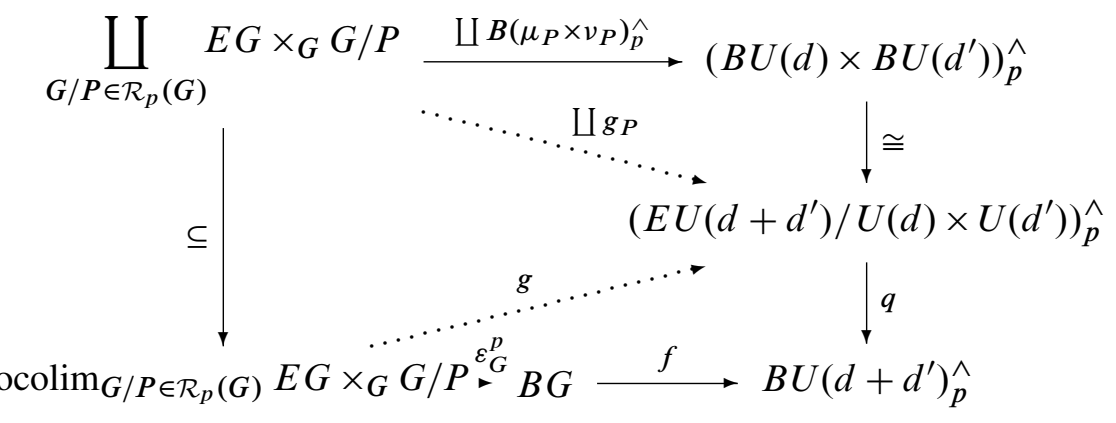

The diagram of solid arrows commutes up to homotopy. Since $q$ is a fibration, we can choose maps

$$
g_{P}: E G \times_{G} G / P \rightarrow\left(E U\left(d+d^{\prime}\right) /\left(U(d) \times U\left(d^{\prime}\right)\right)\right)_{p}^{\wedge}
$$

such that the upper triangle commutes up to homotopy, and the remaining part of the diagram, ie

$$
\coprod_{G / P \in \mathcal{R}_{p}(G)} E G \times_{G} G / P \stackrel{\amalg g_{P}}{\longrightarrow}\left(E U\left(d+d^{\prime}\right) / U(d) \times U\left(d^{\prime}\right)\right)_{p}^{\wedge}
$$

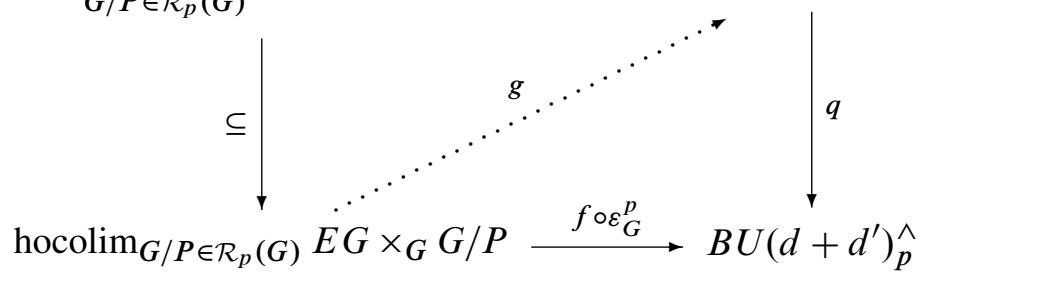

commutes strictly.

Proposition 4.4 If the lifting extension problem (4.3) has a solution, then $\mu$ and $v$ are $p$-homotopy characters.

Proof Let $g$ be a lifting extension. Since $\varepsilon_{G}^{p}$ is a $p$-equivalence, there exists a map $f^{\prime}$ unique up to homotopy such that the composition

$$
\operatorname{hocolim}_{G / P \in \mathcal{R}_{p}(G)} E G \times \times_{G} G / P \stackrel{\varepsilon_{G}^{p}}{\longrightarrow} B G \stackrel{f^{\prime}}{\longrightarrow}\left(E U\left(d+d^{\prime}\right) / U(d) \times U\left(d^{\prime}\right)\right)_{p}^{\wedge}
$$

is homotopic to $g$. The composition

$$
\tilde{f}: B G \stackrel{f^{\prime}}{\longrightarrow}\left(E U\left(d+d^{\prime}\right) / U(d) \times U\left(d^{\prime}\right)\right)_{p}^{\wedge} \stackrel{\simeq}{\longrightarrow}\left(B U(d) \times B U\left(d^{\prime}\right)\right)_{p}^{\wedge}
$$

fits into the diagram of Proposition 4.1, since $T$ is contained in some $p$-stubborn subgroup. Thus the conclusion follows from Proposition 4.1. 


\section{Obstruction theory}

Now our goal is to construct a lifting extension of the diagram (4.3). Let us sketch the argument we use here; the formal proof is given in Sections 7 and 8. In Section 3 we develop the obstruction theory for lifting maps from homotopy colimits; let $\operatorname{Fib}_{\mu, \nu}^{p}: \mathcal{R}_{p}(G) \rightarrow$ Sp be the functor defined in (3.2) which is associated to the diagram (4.3). We need to check that the assumptions which allow to construct this functor are satisfied but it will become clear when we calculate the homotopy type of the spaces $\operatorname{Fib}_{\mu, \nu}^{p}(G / P)$. By Theorem 3.4, the problem (4.3) has a solution if

$$
H^{i+1}\left(\mathcal{R}_{p}(G) ; \pi_{i} \operatorname{Fib}_{\mu, \nu}^{p}\right)=0
$$

for all $i \geq 1$. By Proposition 2.14 it is sufficient to prove that

$$
\Lambda^{i+1}\left(N_{G}(P) / P ; \pi_{i} \operatorname{Fib}_{\mu, \nu}^{p}(G / P)\right)=0
$$

for $G / P \in \mathcal{R}_{p}(G), i \geq 1$. This makes it important to describe the spaces $\operatorname{Fib}_{\mu, v}^{p}(G / P)$ and the action of the groups $N_{G}(P) / P$ on them. For a $p$-stubborn subgroup $P \subseteq G$ the space $\operatorname{Fib}_{\mu, v}^{p}(G / P)$ is, by definition (3.2), the homotopy fiber of the map

$$
\begin{aligned}
\operatorname{map}\left(E G / P,\left(B U(d) \times B U\left(d^{\prime}\right)\right)_{p}^{\wedge}\right)_{B} & \mu_{P} \times B v_{P} \\
& \rightarrow \operatorname{map}\left(E G / P, B U\left(d+d^{\prime}\right)_{p}^{\wedge}\right)_{B\left(\mu_{P} \oplus v_{P}\right)}
\end{aligned}
$$

induced by the inclusion $U(d) \times U\left(d^{\prime}\right) \subseteq U\left(d+d^{\prime}\right)$. By the Dwyer-ZabrodskyNotbohm theorem, this map is homotopy equivalent to

$$
B C_{U(d) \times U\left(d^{\prime}\right)}\left(\mu_{P}(P)\right)_{p}^{\wedge} \times B C_{U(d) \times U\left(d^{\prime}\right)}\left(v_{P}(P)\right)_{p}^{\wedge} \rightarrow B C_{U\left(d+d^{\prime}\right)}\left(\left(\mu_{P} \times v_{P}\right)(P)\right)_{p}^{\wedge},
$$

which is, by Schur's lemma, equivalent to the map

$$
\prod_{\rho \in \operatorname{IrrRep}(P)} B\left(U\left(c_{\mu_{P}}^{\rho}\right) \times U\left(c_{\nu_{P}}^{\rho}\right)\right)_{p}^{\wedge} \rightarrow \prod_{\rho \in \operatorname{IrrRep}(P)} B U\left(c_{\mu_{P}}^{\rho}+c_{\nu_{P}}^{\rho}\right)_{p}^{\wedge},
$$

induced again by the inclusions $U\left(c_{\mu_{P}}^{\rho}\right) \times U\left(c_{\nu_{P}}^{\rho}\right) \subseteq U\left(c_{\mu_{P}}^{\rho}+c_{\nu_{P}}^{\rho}\right)$. Thus there is a homotopy equivalence

$$
\operatorname{Fib}_{\mu, \nu}^{p}(G / P) \simeq \prod_{\rho \in \operatorname{IrRep}(P)}\left(U\left(c_{\mu_{P}}^{\rho}+c_{v_{P}}^{\rho}\right) /\left(U\left(c_{\mu_{P}}^{\rho}\right) \times U\left(c_{v_{P}}^{\rho}\right)\right)\right)_{p}^{\wedge} .
$$

To proceed with calculations we need to describe also the homotopy action of the group $\operatorname{Aut}_{\mathcal{R}_{p}(G / P)}(G / P)=N_{G}(P) / P$ on $\operatorname{Fib}_{\mu, v}^{p}(G / P)$. There is an $N_{G}(P) / P$-action on the right-hand side of the equivalence (4.6) induced by the action on $\operatorname{IrrRep}(P)$. We must show that these actions coincide. 
We will need some definitions and they will be given in somewhat greater generality. Let $P$ be an arbitrary $p$-toral group, $N$ a finite group acting on $P$ by outer automorphisms (ie there is a given homomorphism $N \rightarrow \operatorname{Out}(P)$ ), and $\alpha, \beta: P \rightarrow U(d)$ be homomorphisms which are $N$-invariant up to conjugacy. Define a left $N$-space

$$
\mathfrak{G}_{\alpha, \beta}:=\prod_{\rho \in \operatorname{IrrRep}(P)}\left(U\left(c_{\alpha}^{\rho}+c_{\beta}^{\rho}\right) /\left(U\left(c_{\alpha}^{\rho}\right) \times U\left(c_{\beta}^{\rho}\right)\right)\right)_{p}^{\wedge},
$$

where $N$ acts by permuting factors, ie $\eta\left(\left(x_{\rho}\right)_{\rho \in \operatorname{IrrRep}(P)}\right)=\left(x_{\eta^{*} \rho}\right)_{\rho \in \operatorname{IrrRep}(P)}$ for $\eta \in N$. A homotopy $N$-space is a space $X$ equipped with a homomorphism from $N$ into the group of the homotopy classes of self-homotopy equivalences of $X$; in particular, every $N$-space may be regarded as a homotopy $N$-space. We say that homotopy $N$-spaces $X$ and $Y$ are homotopy $N$-equivalent, see Definition 7.1, if there exists a map $f: X \rightarrow Y$ such that the diagram

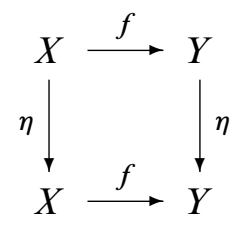

commutes up to homotopy for every $\eta \in N$.

Theorem 4.9 Let $\mu, v$ be $\mathcal{P}$-characters of a compact connected Lie group $G$ such that $\mu+v$ is a homotopy character. For every $p$-stubborn subgroup $P \subseteq G$, the homotopy $N_{G}(P) / P$-spaces $\mathfrak{G}_{\mu_{P}, v_{P}}$ and $\operatorname{Fib}_{\mu, v}^{p}(G / P)$ are homotopy $N_{G}(P) / P$-equivalent.

The proof of this theorem is given at the end of Section 8. This result allows us to prove the following.

Theorem 4.10 Let $\mu, v$ be $\mathcal{P}$-characters of a compact connected Lie group $G$ such that $\mu+v$ is a homotopy character. If for every $p$-stubborn subgroup $P \subseteq G$ and every $i \geq 2$ holds

$$
\Lambda^{i+1}\left(N_{G}(P) / P ; \pi_{i}\left(\mathfrak{G}_{\mu_{P}, \nu_{P}}\right)\right)=0,
$$

then $\mu$ and $v$ are $p$-homotopy characters.

Proof We will apply Theorem 3.4 to prove the existence of the lifting extension of the diagram (4.3). First, we need to check that the assumptions of Theorem 3.4 are satisfied. For every $p$-stubborn subgroup $P \subseteq G$, the group $C_{U\left(d+d^{\prime}\right)}\left(\left(\mu_{P} \times v_{P}\right)(P)\right)$ is connected (by Schur's lemma), and there is a homotopy equivalence

$$
\operatorname{map}\left(E G \times_{G} G / P, B U\left(d+d^{\prime}\right)_{p}^{\wedge}\right)_{B\left(\mu_{P} \times v_{P}\right)} \simeq B C_{U\left(d+d^{\prime}\right)}\left(\left(\mu_{P} \times v_{P}\right)(P)\right)_{p}^{\wedge},
$$

by the Dwyer-Zabrodsky-Notbohm theorem (Theorem 2.1). 
Thus the space

$$
\operatorname{map}\left(E G \times_{G} G / P, B U\left(d+d^{\prime}\right)_{p}^{\wedge}\right)_{B\left(\mu_{P} \times v_{P}\right)}
$$

is simply connected. Also $\operatorname{Fib}_{\mu, \nu}^{p}(G / P)$ is simply connected since it is homotopy equivalent to $\mathfrak{G}_{\mu_{P}, \nu_{P}}$ by Theorem 4.9. Again by Theorem 4.9 and the assumption

$$
\Lambda^{i+1}\left(N_{G}(P) / P ; \operatorname{Fib}_{\mu, \nu}^{p}(G / P)\right)=\Lambda^{i+1}\left(N_{G}(P) / P ; \pi_{i}\left(\mathfrak{G}_{\mu_{P}, \nu_{P}}\right)\right)=0
$$

for $i \geq 2$ and $G / P \in \mathcal{R}_{p}(G)$. The space $\mathfrak{G}_{\mu_{P}, \nu_{P}}$ is simply connected; therefore this equation is valid also for $i=1$. Thus, by Proposition 2.14,

$$
H^{i+1}\left(\mathcal{R}_{p}(G) ; \operatorname{Fib}_{\mu, \nu}^{p}\right)=0
$$

for $i \geq 1$. Theorem 3.4 implies that there exists a lifting extension in the diagram (4.3). The conclusion follows from Proposition 4.4.

\section{Calculation of groups $\Lambda^{*}$}

Proposition 4.11 Let $P \subseteq G$ be a $p$-stubborn subgroup. Assume that for every $N_{G}(P) / P$-orbit $X \subseteq \operatorname{IrrRep}\left(P, \mu_{P}\right) \cap \operatorname{IrrRep}\left(P, v_{P}\right)$ and every $i \geq 2$,

$$
\Lambda^{i+1}\left(N_{G}(P) / P ; \mathbb{Z}_{p}^{\wedge}[X]\right)=0 .
$$

Then $\Lambda^{i+1}\left(N_{G}(P) / P ; \pi_{i}\left(\mathfrak{G}_{\mu_{P}, v_{P}}\right)\right)=0$ for $i \geq 2$.

Proof Denote $N=N_{G}(P) / P, \mathfrak{I}:=\operatorname{IrrRep}\left(P, \mu_{P}\right) \cap \operatorname{IrrRep}\left(P, v_{P}\right)$, and

$$
Y_{\rho}:=\left(U\left(c_{\mu_{P}}^{\rho}+c_{\mu_{P}}^{\rho}\right) /\left(U\left(c_{\mu_{P}}^{\rho}\right) \times U\left(c_{\nu_{P}}^{\rho}\right)\right)\right)_{p}^{\wedge}
$$

for $\rho \in \operatorname{IrrRep}(P)$. Note that for $\rho \in \operatorname{IrrRep}(P) \backslash \mathfrak{I}$ either $c_{\mu_{P}}^{\rho}=0$ or $c_{\nu_{P}}^{\rho}=0$; thus

$$
\mathfrak{G}_{\mu_{P}, v_{P}}=\prod_{\rho \in \mathfrak{I}} Y_{\rho} .
$$

Let $\mathfrak{I}=X_{1} \cup \cdots \cup X_{k}$ be a presentation as the sum of disjoint orbits, and let $\rho_{j} \in X_{j}$ be representatives. Obviously $Y_{\rho}=Y_{\rho^{\prime}}$ if $\rho$ and $\rho^{\prime}$ lie in the same orbit. We have a sequence of isomorphisms of $\mathbb{Z}_{p}^{\wedge}\left[N_{G}(P) / P\right]$-modules:

$$
\begin{aligned}
\pi_{i}\left(\mathfrak{G}_{\mu_{p}, v_{p}}\right) & =\pi_{i}\left(\prod_{\rho \in \mathfrak{I}} Y_{\rho}\right)=\prod_{j=1}^{k} \pi_{i}\left(\prod_{\rho \in X_{j}} Y_{\rho}\right) \\
& =\prod_{j=1}^{k} \pi_{i}\left(Y_{\rho_{j}}^{X_{j}}\right)=\prod_{j=1}^{k} \pi_{i}\left(Y_{\rho_{j}}\right) \otimes \mathbb{Z}_{p}^{\wedge}\left[X_{j}\right]
\end{aligned}
$$


Since $\pi_{i}\left(Y_{\rho_{j}}\right)$ is a finitely generated $\mathbb{Z}_{p}^{\wedge}$-module, from Proposition 2.16 follows that

$$
\begin{aligned}
\Lambda^{i}\left(N ; \pi_{i}\left(\mathfrak{G}_{\mu_{P}, \nu_{P}}\right)\right) & =\Lambda^{i}\left(N ; \prod_{j=1}^{k} \mathbb{Z}_{p}^{\wedge}\left[X_{j}\right] \otimes \pi_{i}\left(Y_{\rho_{j}}\right)\right) \\
& =\bigoplus_{j=1}^{k} \Lambda^{i}\left(N ; \mathbb{Z}_{p}^{\wedge}\left[X_{j}\right] \otimes \pi_{i}\left(Y_{\rho_{j}}\right)\right) \\
& =0 .
\end{aligned}
$$

Proof of Theorem 1.5 The result now follows immediately from Theorem 4.10 and Proposition 4.11.

\section{Splitting of Adams operations}

Finally, we will prove that Theorem 1.5 applies in the case that $G$ is the rank $n$ unitary group, and $v=\psi^{k}$ is the character of the unstable Adams operation of degree $k$, $(k, n !)=1$. The special case is the character of the identity representation $\iota=\psi^{1}$. The proof of Theorem 1.5 depends on the following two propositions. For a finite set $X$ let $\Sigma_{X}$ denote the group of permutations of $X$.

Proposition 4.12 Let $P$ be a $p$-stubborn subgroup of $U(n)$, and let $k$ be an integer prime to $n !$. For every $N_{U(n)}(P) / P$-orbit $X \subseteq \operatorname{IrrRep}\left(P, \psi_{P}^{k}\right)$, the map $N_{U(n)}(P) / P \rightarrow$ $\Sigma_{X}$ induced by this action is an epimorphism.

Proposition 4.13 If $X$ is a finite set, then $\Lambda^{i}\left(\Sigma_{X} ; \mathbb{Z}_{p}^{\wedge}[X]\right)=0$ for $i \geq 2$.

These propositions are proven at the end of Section 5 and Section 6, respectively.

Finally, we prove that the character $\psi^{k}$ of the unstable Adams operation $\Psi^{k}: B U(n) \rightarrow$ $B U(n)$ has the splitting property.

Proof of Theorem 1.4 Let $\mu$ be a $\mathcal{P}$-character of $U(n)$ such that $\mu+\psi^{k}$ is a homotopy character. Fix a prime $p$, a $p$-stubborn subgroup $P \subseteq U(n)$ and an $N_{U(n)}(P) / P$-orbit $X \subseteq \operatorname{IrrRep}\left(P, \psi^{k}\right)$. By Proposition 2.15(2), if $p$ divides the order of $\operatorname{ker}\left(N_{U(n)}(P) / P \rightarrow \Sigma_{X}\right)$, then $\Lambda^{i}\left(N_{U(n)}(P) / P ; \mathbb{Z}_{p}^{\wedge}[X]\right)=0$; otherwise

$$
\Lambda^{i}\left(N_{G}(P) / P ; \mathbb{Z}_{p}^{\wedge}[X]\right)=\Lambda^{i}\left(\Sigma_{X} ; \mathbb{Z}_{p}^{\wedge}[X]\right)=0
$$

for $i \geq 2$ by Propositions 4.12 and 4.13. The conclusion follows from Theorem 1.5. 


\section{Representations of $p$-stubborn subgroups of $U(n)$}

Let $n$ be a positive integer and let $T_{n} \subseteq U(n)$ be a maximal torus. Recall $[13 ; 5 ; 6]$ that for every $m \in \mathbb{Z}$ such that $(m, n !)=1$, there exists a unique homotopy representation $\Psi^{m}: B U(n) \rightarrow B U(n)$ such that $\left.\Psi^{m}\right|_{B T_{n}}$ is a map induced by the composition

$$
T_{n} \rightarrow T_{n} \subseteq U(n), \quad x \mapsto x^{m} .
$$

Let $\psi^{m} \in R(U(n))$ be the character of $\Psi^{m}$, and let $p$ be a fixed prime integer. In this section we prove Proposition 4.12, which states that for every $p$-stubborn subgroup $P \subseteq U(n)$ and every $N_{U(n)}(P) / P$-orbit $X \subseteq \operatorname{IrrRep}\left(P, \psi_{P}^{m}\right)$, the map $N_{U(n)}(P) / P \rightarrow \Sigma_{X}$ is surjective.

Let us recall the classification of $p$-stubborn subgroups of unitary groups obtained by Oliver [12]. Define $p \times p$-matrices

$$
A=\left(\begin{array}{ccccc}
1 & 0 & 0 & \cdots & 0 \\
0 & \zeta & 0 & \cdots & 0 \\
0 & 0 & \zeta^{2} & \cdots & 0 \\
\vdots & \vdots & \vdots & \ddots & \vdots \\
0 & 0 & 0 & \cdots & \zeta^{p-1}
\end{array}\right), \quad B=\left(\begin{array}{ccccc}
0 & 1 & 0 & \cdots & 0 \\
0 & 0 & 1 & \cdots & 0 \\
\vdots & \vdots & \vdots & \ddots & \vdots \\
0 & 0 & 0 & \cdots & 1 \\
1 & 0 & 0 & \cdots & 0
\end{array}\right)
$$

where $\zeta=e^{2 \pi i / p}$. Let $I_{k}$ denote the $k \times k$ identity matrix. Define matrices $A_{i}^{k}, B_{i}^{k} \in$ $U\left(p^{k}\right)$, for $i=0, \ldots, k-1$, by

$$
A_{i}^{k}=I_{p^{i}} \otimes A \otimes I_{p^{k-i-1}}, \quad B_{i}^{k}=I_{p^{i}} \otimes B \otimes I_{p^{k-i-1}} .
$$

Finally, let

$$
\Gamma(k):=\left\langle S^{1}, A_{0}^{k}, \ldots, A_{k-1}^{k}, B_{0}^{k}, \ldots, B_{k-1}^{k}\right\rangle \subseteq U\left(p^{k}\right),
$$

where $S^{1}=\left\langle e^{2 \pi i t} \cdot I_{p^{k}}\right\rangle$. Note that $\left(A_{i}^{k}\right)^{p}=\left(B_{i}^{k}\right)^{p}=I_{p^{k}}$ and

$$
A_{i}^{k} A_{j}^{k}=A_{j}^{k} A_{i}^{k}, \quad B_{i}^{k} B_{j}^{k}=B_{j}^{k} B_{i}^{k}, \quad B_{i}^{k} A_{j}^{k}= \begin{cases}\zeta \cdot A_{j}^{k} B_{i}^{k} & \text { for } i=j, \\ A_{j}^{k} B_{i}^{k} & \text { for } i \neq j .\end{cases}
$$

Furthermore, every element of $x \in \Gamma(k)$ can be written uniquely in the form

$$
x=t \cdot A_{i_{1}}^{k} \cdots A_{i_{r}}^{k} B_{j_{1}}^{k} \cdots B_{j_{s}}^{k},
$$

where $t \in S^{1}, 0 \leq i_{1}<\cdots<i_{r}<k$, and $0 \leq j_{1}<\cdots<j_{s}<k$.

Let $C_{p}$ denote the cyclic group of order $p$. We say that a subgroup of a unitary group is elementary $p$-stubborn if it has the form

$$
\Gamma\left(k ; a_{1}, \ldots, a_{r}\right):=\Gamma(k) \curlywedge C_{p}^{a_{1}} \prec \cdots \prec C_{p}^{a_{r}} \subseteq U\left(p^{k}\right) \curlywedge C_{p}^{a_{1}} \prec \cdots \prec C_{p}^{a_{r}} \subseteq U\left(p^{k+\sum a_{i}}\right) .
$$


If $P=\Gamma\left(k ; a_{1}, \ldots, a_{r}\right)$ is an elementary $p$-stubborn subgroup, then by [12, Theorem 6]

$$
N_{U(n)}(P) / P \simeq \operatorname{Sp}_{2 k}\left(\mathbb{F}_{p}\right) \times \operatorname{GL}_{a_{1}}\left(\mathbb{F}_{p}\right) \times \cdots \times \mathrm{GL}_{a_{r}}\left(\mathbb{F}_{p}\right) .
$$

Proposition 5.6 [12, Theorem 8] Every p-stubborn subgroup of $U(n)$ is conjugate to

$$
P=P_{1}^{b_{1}} \times \cdots \times P_{j}^{b_{j}} \subseteq U\left(p^{n_{1}}\right)^{b_{1}} \times \cdots \times U\left(p^{n_{j}}\right)^{b_{j}} \subseteq U(n),
$$

where $\left\{P_{i} \subseteq U\left(p^{n_{i}}\right)\right\}$ is a family of pairwise nonisomorphic elementary $p$-stubborn subgroups. Furthermore,

$$
N_{U(n)}(P) / P \simeq \prod_{i=1}^{j}\left(N_{U\left(p^{n_{i}}\right)}\left(P_{i}\right) / P_{i}\right) \prec \Sigma_{b_{i}} .
$$

Recall that $\psi^{m}$ denotes the character of the unstable Adams operation of degree $m$. For an arbitrary subgroup $P \subseteq U(n)$, the homomorphism $\psi_{P}^{m}$ is determined by the condition that for all $x \in P$,

$$
\operatorname{tr}\left(\psi_{P}^{m}(x)\right)=\operatorname{tr}\left(x^{m}\right)
$$

uniquely up to conjugacy. This follows from the definition of $\Psi^{m}$, since every $x \in P$ is conjugate to an element of $T_{n}$.

Proposition 5.9 If $n=p^{k}$, then the representation $\psi_{\Gamma(k)}^{m}$ is irreducible.

Proof If $k=0$, then $\psi_{\Gamma(k)}^{m}$ has dimension 1. Otherwise, $p$ does not divide $m$. Define an endomorphism $\alpha: \Gamma(k) \rightarrow \Gamma(k)$ by formulas

- $\alpha(x)=x^{m}$ for $x \in S^{1} \cong Z\left(U\left(p^{k}\right)\right)$

- $\alpha\left(A_{i}^{k}\right)=\left(A_{i}^{k}\right)^{m}$

- $\alpha\left(B_{i}^{k}\right)=B_{i}^{k}$.

By checking conditions (5.4) we prove that $\alpha$ is well-defined, and it is surjective since $(p, m)=1$. Let

$$
x=t \cdot A_{i_{1}}^{k} \cdots A_{i_{r}}^{k} B_{j_{1}}^{k} \cdots B_{j_{s}}^{k} \in \Gamma(k)
$$

be the presentation (5.5) of an arbitrary element of $\Gamma(k)$. If $s=0$, then $\alpha(x)=x^{m}$; for $s>0$ both $x^{m}$ and $\alpha^{m}(x)$ have no nonzero elements on the diagonal, and then $\operatorname{tr}\left(x^{m}\right)=\operatorname{tr}(\alpha(x))=0$. Therefore, for every $x \in \Gamma(k)$ we have $\operatorname{tr}\left(\psi_{\Gamma(k)}^{m}(x)\right)=\operatorname{tr}\left(x^{m}\right)$, and then the composition $\iota_{\Gamma(k)} \circ \alpha$ is conjugate to $\psi_{\Gamma(k)}^{m}$ since they have equal characters. Now the conclusion follows from irreducibility of $\iota_{\Gamma(k)}$, which is proven in the proof of [12, Theorem 6], and surjectivity of $\alpha$. 
Proposition 5.10 For every elementary $p$-stubborn subgroup $P$ of $U(n)$, the representation $\psi_{P}^{m}$ is irreducible.

Proof We induct with respect to $j$, where $P=\Gamma\left(k ; a_{1}, \ldots, a_{j}\right)$. The case $j=0$ is proven in Proposition 5.9. Assume that $j>0$. We denote $n^{\prime}:=n / p^{a_{j}}$,

$$
Q:=\Gamma\left(k ; a_{1}, \ldots, a_{j-1}\right) \subseteq U\left(n^{\prime}\right)
$$

and $E=C_{p}^{a_{j}}$; obviously $P=Q \imath E=Q^{E} \rtimes E$. For $\left(x_{r}\right)_{r \in E} \in Q^{E}$, we have

$$
\begin{aligned}
\operatorname{tr}\left(\psi_{P}^{m}\left(\left(x_{r}\right)_{r \in E}\right)\right. & =\operatorname{tr}\left(\left(x_{r}^{m}\right)_{r \in E}\right)=\sum_{r \in E} \operatorname{tr}\left(x_{r}^{m}\right)=\sum_{r \in E} \operatorname{tr}\left(\psi_{Q}^{m}\left(x_{r}\right)\right) \\
& =\sum_{r \in E} \operatorname{tr}\left(\left(\psi_{Q}^{m} \circ \operatorname{pr}_{r}\right)\left(\left(x_{s}\right)_{s \in E}\right)\right) \\
& =\operatorname{tr}\left(\left(\bigoplus_{r \in E} \psi_{Q}^{m} \circ \operatorname{pr}_{r}\right)\left(\left(x_{s}\right)_{s \in E}\right)\right),
\end{aligned}
$$

where $\mathrm{pr}_{r}$ denotes the projection on the $r^{\text {th }}$ factor. Thus, we have

$$
\operatorname{res}_{Q^{E}}^{P} \psi_{P}^{m} \cong \bigoplus_{r \in E} \psi_{Q}^{m} \circ \operatorname{pr}_{r}
$$

as representations of $Q^{E}$. By the induction hypothesis $\psi_{Q}^{m}$ is irreducible, and then also $\psi_{Q}^{m} \circ \operatorname{pr}_{r}$ is irreducible. The conjugation action of the quotient $P / Q^{E} \cong E$ on $Q^{E}$ is by shifting coordinates. For $t \in E$, we have

$$
\begin{aligned}
\left(t^{*}\left(\psi_{Q}^{m} \circ \mathrm{pr}_{r}\right)\right)\left(\left(x_{s}\right)_{s \in E}\right) & =\left(\psi_{Q}^{m} \circ \mathrm{pr}_{r}\right)\left(\left(x_{s+t}\right)_{s \in E}\right)=\psi_{Q}^{m}\left(x_{r+t}\right) \\
& =\left(\psi_{Q}^{m} \circ \mathrm{pr}_{r+t}\right)\left(\left(x_{s}\right)_{s \in E}\right) .
\end{aligned}
$$

Then $t^{*}\left(\psi_{Q}^{m} \circ \operatorname{pr}_{r}\right)=\psi_{Q}^{m} \circ \operatorname{pr}_{r+t}$, and this implies that $E$ acts transitively on the summands at the right-hand side of equation $(* *)$. Hence the representation induced from any single summand is irreducible, and by Frobenius reciprocity it is isomorphic to $\psi_{P}^{m}$.

Proof of Proposition 4.12 By Proposition 5.6, we can assume that $P=\prod_{i=1}^{j} P_{i}^{b_{i}}$, where $P_{i} \subseteq U\left(p^{r_{i}}\right)$ are pairwise nonisomorphic elementary $p$-stubborn subgroups. For $i=1, \ldots, j$ and $k=1, \ldots, b_{i}$, define

$$
\gamma_{i, k}=\psi_{P_{i}}^{m} \circ \operatorname{pr}_{i, k}
$$


where $\operatorname{pr}_{i, k}: P \rightarrow P_{i}$ is the projection onto the $k^{\text {th }}$ summand of type $P_{i}$. By a calculation similar to $(*)$ in the proof of Proposition 5.10, we prove that

$$
\psi_{P}^{m} \simeq \bigoplus_{i=1}^{j} \bigoplus_{k=1}^{b_{i}} \gamma_{i, k} .
$$

The representations $\gamma_{i, k}$ are pairwise nonisomorphic and irreducible by Proposition 5.10, therefore $\operatorname{IrRep}\left(P, \psi_{P}^{m}\right)=\left\{\gamma_{i, k}\right\}_{i=1, \ldots, j}^{k=1, \ldots, b_{i}}$. Let

$$
p_{i}: N_{U(n)}(P) / P \rightarrow\left(N_{U\left(n_{i}\right)}\left(P_{i}\right) / P_{i}\right)\left\{\Sigma_{b_{i}} \rightarrow \Sigma_{b_{i}} \simeq \Sigma_{\left\{\gamma_{i, k}\right\}_{k=1}^{b_{i}}}\right.
$$

be the homomorphism given by the projection on the $i^{\text {th }}$ summand of (5.7). For every $\eta \in N_{P}(P) / P, i \in\{1, \ldots, j\}$, and $k \in\left\{1, \ldots, b_{i}\right\}$, there exists $\lambda(\eta, i, k) \in N\left(P_{i}\right) / P_{i}$ such that $\mathrm{pr}_{i, k} \circ \eta=\lambda(\eta, i, k) \circ \mathrm{pr}_{i, p_{i}(\eta)(k)}$. Furthermore, for every $\lambda \in N\left(P_{i}\right) / P_{i}$, we have $\lambda^{*} \psi_{P_{i}}^{m}=\psi_{P_{i}}^{m} \circ \lambda=\psi_{P_{i}}^{m}$ by (5.8). Finally, we obtain

$$
\begin{aligned}
\eta^{*} \gamma_{i, k} & =\gamma_{i, k} \circ \eta=\psi_{P_{i}}^{m} \circ \operatorname{pr}_{i, k} \circ \eta=\psi_{P_{i}}^{m} \circ \lambda(\eta, i, k) \circ \operatorname{pr}_{i, p_{i}(\eta)(k)} \\
& =\psi_{P_{i}}^{m} \circ \operatorname{pr}_{i, p_{i}(\eta)(k)}=\gamma_{i, p_{i}(\eta)(k)}
\end{aligned}
$$

for arbitrary $\eta, i, k$. Thus every $N_{U(n)}(P) / P$-orbit of $\operatorname{IrRep}\left(P, \psi_{P}^{m}\right)$ has the form $\left\{\gamma_{i, k}\right\}_{k=1}^{b_{i}}$. The conclusion follows from the surjectivity of the homomorphisms $p_{i}$.

\section{Calculation of functors $\Lambda^{*}$}

Fix a prime $p$. Let $X$ be a finite set and let $\Sigma_{X}$ be the group of permutations of $X$. In this section we prove Proposition 4.13 , which states that $\Lambda^{i}\left(\Sigma_{X} ; \mathbb{Z}_{p}^{\wedge}[X]\right)=0$ for $i \geq 2$. To achieve this, we use results of Aschbacher, Kessar and Oliver [2]. For a finite group $H$, let $O_{p}(H)$ denote the maximal normal $p$-subgroup of $H$. Note that a $p$-subgroup $P \subseteq H$ is $p$-radical if and only if $P=O_{p}\left(N_{H}(P)\right)$.

Definition 6.1 Let $\Gamma$ be a finite group. A radical $p$-chain of length $k$ in $\Gamma$ is a sequence

$$
P_{0} \subsetneq P_{1} \subsetneq P_{2} \subsetneq \cdots \subsetneq P_{k} \subseteq \Gamma
$$

of distinct $p$-subgroups of $\Gamma$ such that

- $P_{0}=O_{p}(\Gamma)$,

- $P_{i}=O_{p}\left(N_{\Gamma}\left(P_{0}\right) \cap N_{\Gamma}\left(P_{1}\right) \cap \cdots \cap N_{\Gamma}\left(P_{i}\right)\right)$,

- $\quad P_{k}$ is a $p$-Sylow subgroup of $N_{\Gamma}\left(P_{0}\right) \cap N_{\Gamma}\left(P_{1}\right) \cap \cdots \cap N_{\Gamma}\left(P_{k-1}\right)$. 
Lemma 6.2 Fix a finite group $\Gamma$, and a finitely generated $\mathbb{Z}_{p}^{\wedge}[\Gamma]$-module $M$. Assume, for some $k \geq 1$, that $\Lambda^{k}(\Gamma ; M) \neq 0$. Then there is a radical $p$-chain

$$
1=P_{0} \subsetneq P_{1} \subsetneq P_{2} \subsetneq \cdots \subsetneq P_{k} \subseteq \Gamma
$$

such that $M / p M$, regarded as an $\mathbb{F}_{p}\left[P_{k}\right]$-module, contains a copy of the free module $\mathbb{F}_{p}\left[P_{k}\right]$.

Proof This is a special case of [2, Lemma 5.27].

Proposition 6.3 Assume that $X$ is a finite set of cardinality $n$ and that $P \subseteq \Sigma_{X}$ is a $p$-radical subgroup. Then

$$
|P| \geq n-\left|X^{P}\right|=\left|X \backslash X^{P}\right| .
$$

Proof Let $\mathfrak{S}$ be the set of all finite (possibly empty) sequences of positive integers. For $\boldsymbol{k}=\left(k_{1}, \ldots, k_{r}\right) \in \mathfrak{S}$, inductively define subgroups $A(\boldsymbol{k}) \subseteq \Sigma_{p^{|\boldsymbol{k}|}},|\boldsymbol{k}|:=k_{1}+\cdots+k_{r}$, by $A(\varnothing)=1 \subseteq \Sigma_{1}$ and

$$
A\left(k_{1}, \ldots, k_{r}\right)=A\left(k_{1}, \ldots, k_{r-1}\right) \curlywedge C_{p}^{k_{r}} \subseteq \Sigma_{p^{k_{1}+\cdots+k_{r-1}}} \prec C_{p}^{k_{r}} \subseteq \Sigma_{p^{|k|}} .
$$

By [1], every $p$-radical subgroup of $\Sigma_{X}$ has the form

$$
P=\prod_{\boldsymbol{k} \in \mathfrak{S}} A(\boldsymbol{k})^{m(\boldsymbol{k})} \subseteq \prod_{\boldsymbol{k} \in \mathfrak{S}}\left(\Sigma_{p^{|\boldsymbol{k}|}}\right)^{m(\boldsymbol{k})} \subseteq \Sigma_{n} \simeq \Sigma_{X},
$$

where $n=\sum m(\boldsymbol{k}) p^{|\boldsymbol{k}|}$. Note that $|A(\boldsymbol{k})| \geq p^{|\boldsymbol{k}|}$ and $A(\varnothing)$ is a trivial group. Thus

$$
|P|=\prod_{\boldsymbol{k} \in \mathfrak{S} \backslash\{\varnothing\}}|A(\boldsymbol{k})|^{m(\boldsymbol{k})} \geq \prod_{\boldsymbol{k} \in \mathfrak{S} \backslash\{\varnothing\}} p^{|\boldsymbol{k}| \cdot m(\boldsymbol{k})} \geq \prod_{\boldsymbol{k} \in \mathfrak{S} \backslash\{\varnothing\}} m(\boldsymbol{k}) \cdot p^{|\boldsymbol{k}|}=n-m(\varnothing) .
$$

Since $\left|X^{P}\right|=m(\varnothing)$, the conclusion follows.

Proposition 6.4 Let $X$ be a finite set with $n$ elements. Assume that

$$
1=P_{0} \subsetneq P_{1} \subsetneq P_{2} \subsetneq \cdots \subsetneq P_{k} \subseteq \Sigma_{X}
$$

is a radical $p$-chain of length $k \geq 2$. Then either $\left|P_{k}\right|>n$, or $p=2, n=5$ and this chain is conjugate to

$$
1=P_{0} \subsetneq P_{1}=\langle a\rangle \subsetneq P_{2}=\langle a, b\rangle \subseteq \Sigma_{X},
$$

where $a, b \in \Sigma_{X}$ are disjoint cycles of length 2 . 
Proof We can assume that $n \geq p^{2}$; otherwise all $p$-subgroups of $\Sigma_{X}$ had orders no more than $p$. Denote $X_{i}=X^{P_{i}}, Y_{i}=X \backslash X_{i}$; obviously $X_{0}=X$. As a consequence of the definition of radical $p$-chain (see, for example, [2]), $P_{i} / P_{i-1}$ is a $p$-radical subgroup of the group

$$
\left(\bigcup_{j=0}^{i-1} N_{\Sigma_{X}}\left(P_{j}\right)\right) / P_{i-1}=\left(\bigcup_{j=0}^{i-1} N_{\Sigma_{Y_{i-1}}}\left(P_{j}\right)\right) / P_{i-1} \times \Sigma_{X_{i-1}} .
$$

Hence, by [5, Theorem 1.6(ii)] it is a product $H \times H^{\prime}$ of $p$-radical subgroups

$$
H \subseteq\left(\bigcup_{j=0}^{i-1} N_{\Sigma_{Y_{i-1}}}\left(P_{j}\right)\right) / P_{i-1} \quad \text { and } \quad H^{\prime} \subseteq \Sigma_{X_{i-1}} .
$$

Clearly $X_{i}=X_{i-1}^{H^{\prime}}$ and by Proposition $6.3\left|P_{i}: P_{i-1}\right| \geq\left|H^{\prime}\right| \geq\left|X_{i-1} \backslash X_{i}\right|$. Thus

$$
\left|P_{k}\right|=\prod_{i=1}^{k}\left|P_{i}: P_{i-1}\right| \geq \prod_{i=1}^{k} \max \left\{p,\left|X_{i-1} \backslash X_{i}\right|\right\} .
$$

Since $\sum_{i=1}^{k}\left|X_{i-1} \backslash X_{i}\right|=\left|X_{0} \backslash X_{k}\right|>n-p, P_{k}>n$ unless $p=2$ and $n \in\{4,5\}$. But the case $n=4$ is excluded, since $\Sigma_{4}$ is not 2-reduced, and the only possibility for $n=5$ is stated above.

Proof of Proposition 4.13 Let $X=\left\{x_{1}, \ldots, x_{n}\right\}$. Assume that $\Lambda^{i}\left(\Sigma_{X} ; \mathbb{Z}_{p}^{\wedge}[X]\right) \neq 0$ for some $i \geq 2$. By Lemma 6.2, there exists a radical $p$-chain $P_{0} \subsetneq \ldots \subsetneq P_{i}$ in $\Sigma_{n}$ such that $\mathbb{F}_{p}[X]$ contains a copy of the free module $\mathbb{F}_{p}\left[P_{2}\right]$. By Proposition 6.4 and dimensional reasons, $(i, p, n)=(2,2,5)$, and $P_{2} \subseteq \Sigma_{5}$ is a subgroup generated by two disjoint cycles of length 2 . But then $\operatorname{dim}\left(\mathbb{F}_{p}\left[P_{2}\right]{ }^{P_{2}}\right)=1$ and $\operatorname{dim}\left(\mathbb{F}_{p}[X]^{P_{2}}\right)=3$ which is impossible, since $\operatorname{dim}\left(\mathbb{F}_{p}[X]\right)-\operatorname{dim}\left(\mathbb{F}_{p}\left[P_{2}\right]\right)=1$.

\section{Homotopy actions}

Let Rep be the category of compact Lie groups and homomorphisms up to conjugacy, ie

$$
\operatorname{Mor}_{\operatorname{Rep}}(G, H):=\operatorname{Hom}(G, H) / \operatorname{Inn}(H) .
$$

Fix a finite group $N$; it will be regarded as the category with a single object with $N$ as the group of automorphisms.

Definition 7.1 A left (resp. right) homotopy $N$-action (or an h- $N$-action in short) on a compact Lie group $\Gamma$ is a functor $N \rightarrow \operatorname{Rep}\left(\right.$ resp. $N^{\text {op }} \rightarrow \operatorname{Rep}$ ) whose value on the 
single object of $N$ is $\Gamma$. Similarly, a left (resp. right) h- $N$-action on a space $X$ is a functor $N \rightarrow$ HSp (resp. $N^{\text {op }} \rightarrow$ HSp) with value $X$. A group $\Gamma$ (resp. a space $X$ ) equipped with a left (resp. right) h- $N$-action will be called a left h- $N$-group (resp. right h- $N-$ group, left h- $N$-space, right h- $N$-space). Let $N-\operatorname{Rep}$ (resp. $N^{\text {op }}-\mathbf{R e p}, N-\mathbf{H S p}$, $N^{\text {op }}$-HSp) denote the category of left h- $N$-groups (resp. right h- $N$-groups, left h- $N$ spaces, right h- $N$-spaces), where morphisms are natural transformations of functors. Either a homomorphism or a map is called h- $N$-equivariant (resp. h- $N$-equivalence) if it represents a morphism (resp. an isomorphism) in the suitable category, ie if it preserves an $\mathrm{h}-\mathrm{N}$-action.

Note that a map between h- $N$-spaces is an h- $N$-equivalence if and only if it is both $\mathrm{h}-N$-equivariant and a homotopy equivalence. The classifying space functor maps conjugate homomorphisms into homotopic maps. Thus, it defines functors

$$
B: N-\operatorname{Rep} \rightarrow N-\mathbf{H S p} \quad \text { and } \quad B: N^{\mathrm{op}}-\operatorname{Rep} \rightarrow N^{\mathrm{op}}-\mathbf{H S p} .
$$

Definition 7.2 Let $K$ be a left h- $N$-group and let $H$ be a compact Lie group. We say that a homomorphism $\alpha: K \rightarrow H$ is h- $N$-invariant if for every $\eta \in N$ the representations $[\alpha]$ and $[\alpha] \circ \eta$ are equal (as morphisms in Rep).

\section{Functoriality of centralizers}

Let $H$ be a compact Lie group, $P$ a $p$-toral left h- $N$-group, and $\alpha: P \rightarrow H$ an h-Ninvariant homomorphism. For an arbitrary homomorphism $\gamma: P \rightarrow P$ representing the image of $\eta \in N$ in $\operatorname{Out}(N)$, there exists $b_{\gamma} \in H$ such that the diagram

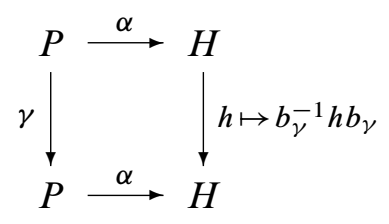

is strictly commutative. The formula

$$
C_{H}(\alpha(P)) \rightarrow C_{H}(\alpha(P)), \quad h \mapsto b_{\gamma} h b_{\gamma}^{-1}
$$

defines a homomorphism which does not depend, up to conjugacy, on the choice of a representative $\gamma$. To prove the independence (up to conjugacy again) on the choice of element $b_{\gamma}$, fix another $b_{\gamma}^{\prime} \in H$ such that $\left(b_{\gamma}^{\prime}\right)^{-1} \alpha(g) b_{\gamma}^{\prime}=\alpha(\gamma(g))$ for all $g \in P$. It is enough to prove that $b_{\gamma}^{\prime} b_{\gamma}^{-1} \in C_{H}(\alpha(P))$, which follows from this calculation:

$\left(b_{\gamma}^{\prime} b_{\gamma}^{-1}\right)^{-1} \alpha(g)\left(b_{\gamma}^{\prime} b_{\gamma}^{-1}\right)=b_{\gamma}\left(\left(b_{\gamma}^{\prime}\right)^{-1} \alpha(g) b_{\gamma}^{\prime}\right) b_{\gamma}^{-1}=b_{\gamma}\left(\left(b_{\gamma}\right)^{-1} \alpha(g) b_{\gamma}\right) b_{\gamma}^{-1}=\alpha(g)$.

Thus, (7.4) defines a right h- $N$-action on $C_{H}(\alpha(P))$. 
Proposition 7.5 Let $P$ be a $p$-toral left h- $N$-group, $H$ a compact Lie group and $\alpha: P \rightarrow H$ an h- $N$-invariant homomorphism. The map (see Theorem 2.1)

$$
\left(\operatorname{ad}_{\alpha}\right)_{p}^{\wedge}: B C_{H}(\alpha(P))_{p}^{\wedge} \rightarrow\left(\operatorname{map}(B P, B H)_{B \alpha}\right)_{p}^{\wedge}
$$

is an equivalence of right $\mathrm{h}-N$-spaces.

Proof We have to prove that for every $\eta \in N$, and every $\gamma: P \rightarrow P$ representing the image of $\eta \in N$ in $\operatorname{Out}(N)$, the following diagram commutes up to homotopy.

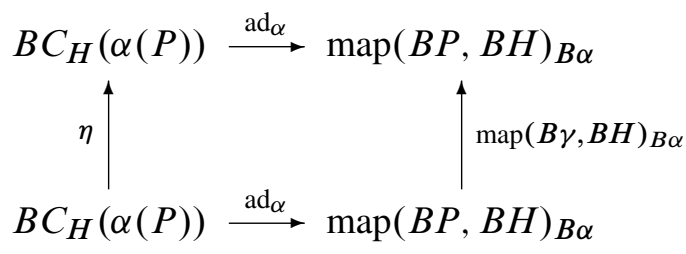

After passing to adjoint maps, this reduces to checking that maps

$$
B \varphi, B \psi: B\left(C_{H}(\alpha(P)) \times P\right) \rightarrow B H
$$

are homotopic, where

$$
\begin{array}{ll}
\varphi: C_{H}(\alpha(P)) \times P \rightarrow H, & \left(g, g^{\prime}\right) \mapsto b_{\gamma} g b_{\gamma}^{-1} \cdot \alpha\left(g^{\prime}\right), \\
\psi: C_{H}(\alpha(P)) \times P \rightarrow H, & \left(g, g^{\prime}\right) \mapsto g \cdot \alpha\left(\gamma\left(g^{\prime}\right)\right),
\end{array}
$$

and $b_{\gamma} \in H$ is an element such that $b_{\gamma}^{-1} \cdot \alpha(g) b_{\gamma}=\alpha(\gamma(g))$ for all $g \in P$. Since $\varphi$ and $\psi$ are conjugate, they induce homotopic maps. Thus $\operatorname{ad}_{\alpha}$ preserves the $\mathrm{h}-N$-action, and by Theorem $2.1\left(\operatorname{ad}_{\alpha}\right)_{p}^{\wedge}$ is a homotopy equivalence; therefore it is an equivalence of right h- $N$-spaces.

\section{Centralizers in unitary groups}

At this point we restrict to the case $H=U(d)$. As before, $P$ is a $p$-toral left h- $N$-group and $\alpha: P \rightarrow U(d)$ is an h- $N$-invariant homomorphism.

Recall that $\operatorname{IrrRep}(P)$ denotes the set of isomorphism classes of unitary representations of $P$ and, for $\rho \in \operatorname{IrrRep}(P)$ and a representation $\xi$ of $P, c_{\xi}^{\rho}$ denotes the multiplicity of $\rho$ in $\xi$, ie the number of summands isomorphic to $\rho$ in an arbitrary decomposition of $\xi$ into a sum of irreducible representations. Define a group

$$
J_{\alpha}=\prod_{\rho \in \operatorname{IrrRep}(P)} U\left(c_{\alpha}^{\rho}\right) .
$$

The formula

$$
\eta: J_{\alpha} \rightarrow J_{\alpha}, \quad\left(f_{\rho}\right)_{\rho \in \operatorname{IrrRep}(P)} \mapsto\left(f_{\eta^{*} \rho}\right)_{\rho \in \operatorname{IrrRep}(P)},
$$


for $\eta \in N$, defines a (strict) right $N$-action on $J_{\alpha}$. In particular, $J_{\alpha}$ is a right h- $N$-group.

The homomorphism $\alpha$ defines a linear $P$-action on $\mathbb{C}^{d}$. For $\rho \in \operatorname{IrrRep}(P)$, let $W_{\rho} \subseteq \mathbb{C}^{d}$ be the maximal subrepresentation with all irreducible summands isomorphic to $\rho$. By Schur's lemma, we have a decomposition

$$
\mathbb{C}^{d} \simeq \bigoplus_{\rho \in \operatorname{IrrRep}(P)} W_{\rho}
$$

An element $\varphi \in U(d)$ centralizes $\alpha(P)$ if and only if for every $\rho \in \operatorname{IrrRep}(P)$ the restriction $\left.\varphi\right|_{W_{\rho}}$ is a $P$-equivariant automorphism of $W_{\rho}$. Then the homomorphism

$$
\prod_{\rho \in \operatorname{IrrRep}(P)} U_{P}\left(W_{\rho}\right) \rightarrow C_{U(d)}(\alpha(P))
$$

is an isomorphism, where $U_{P}\left(W_{\rho}\right)$ is the group of $P$-equivariant unitary automorphisms of $W_{\rho}$. For any $\rho \in \operatorname{IrrRep}(P)$, let $V_{\rho}$ be a $P$-vector space which represents $\rho$ and let $i_{\rho}: V_{\rho} \otimes \mathbb{C}^{c_{\alpha}^{\rho}} \rightarrow W_{\rho}$ be a fixed $P$-isomorphism. The homomorphism

$$
k_{\alpha}^{\rho}: U\left(c_{\alpha}^{\rho}\right) \rightarrow U_{P}\left(W_{\rho}\right), \quad f \mapsto i_{\rho} \circ\left(\mathrm{id}_{V_{\rho}} \otimes f\right) \circ i_{\rho}^{-1}
$$

is an isomorphism by Schur's lemma. Finally, define an isomorphism

$$
k_{\alpha}: J_{\alpha}=\prod_{\rho \in \operatorname{IrRRep}(P)} U\left(c_{\alpha}^{\rho}\right) \stackrel{\oplus k_{\alpha}^{\rho}}{\longrightarrow} \prod_{\rho \in \operatorname{IrrRep}(P)} U_{P}\left(W_{\rho}\right) \stackrel{\cong}{\longrightarrow} C_{U(d)}(\alpha(P)) .
$$

Proposition 7.10 The homomorphism $k_{\alpha}: J_{\alpha} \rightarrow C_{U(d)}(\alpha(P))$ is an equivalence of right $\mathrm{h}-N$-groups.

Proof Let $\gamma: P \rightarrow P$ be an isomorphism that represents the action of $\eta \in N$ on $P$, and let $b_{\gamma} \in U(d)$ be an element such that $b_{\gamma}^{-1} \alpha(g) b_{\gamma}=\alpha(\gamma(g))$ for $g \in P$; see (7.3). By Schur's lemma, $b_{\gamma}$ maps $W_{\eta^{*} \rho}$ isomorphically onto $W_{\rho}$ for every $\rho \in \operatorname{IrrRep}(P)$. Then there is a presentation

$$
b_{\gamma}=\bigoplus_{\rho \in \operatorname{IrrRep}(P)} b_{\gamma}^{\rho},
$$

where $b_{\gamma}^{\rho}: W_{\eta^{*} \rho} \rightarrow W_{\rho}$ are isomorphisms. The diagram

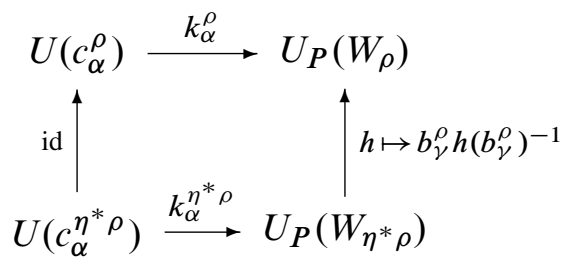


commutes up to conjugacy since both compositions are isomorphisms between unitary groups that preserve subgroups of homotheties. After taking the product over all $\rho \in \operatorname{IrrRep}(P)$ we obtain the diagram

$$
\begin{aligned}
& J_{\alpha} \longrightarrow \prod_{\rho \in \operatorname{IrrRep}(P)} U\left(c_{\alpha}^{\rho}\right) \stackrel{k_{\alpha}}{\longrightarrow} \prod_{\rho \in \operatorname{IrrRep}(P)} U_{P}\left(W_{\rho}\right) \cong C_{U(d)}(\alpha(P)) \\
& \left(f_{\rho}\right) \mapsto\left(f_{\eta^{*} \rho}\right) \uparrow \quad \Pi\left(h \mapsto b_{\gamma}^{\rho} h\left(b_{\gamma}^{\rho}\right)^{-1}\right) \uparrow \\
& J_{\alpha} \longrightarrow \prod_{\rho \in \operatorname{IrrRep}(P)} U\left(c_{\alpha}^{\rho}\right) \stackrel{k_{\alpha}}{\longrightarrow} \prod_{\rho \in \operatorname{IrrRep}(P)} U_{P}\left(W_{\rho}\right) \stackrel{\cong}{\longrightarrow} C_{U(d)}(\alpha(P))
\end{aligned}
$$

which also commutes up to conjugacy. The conclusion follows.

Corollary 7.11 The composition

$$
\left(B J_{\alpha}\right)_{p}^{\wedge} \stackrel{B\left(k_{\alpha}\right)_{p}^{\wedge}}{\longrightarrow} B C_{U(d)}(\alpha(P))_{p}^{\wedge} \stackrel{\left(\operatorname{ad}_{\alpha}\right)_{p}^{\wedge}}{\longrightarrow}\left(\operatorname{map}(B P, B U(d))_{B \alpha}\right)_{p}^{\wedge}
$$

is an equivalence of right $\mathrm{h}-N$-spaces.

Proof This is an immediate corollary to Proposition 7.5 and Proposition 7.10.

\section{Centralizers in products of unitary groups}

Let $\alpha: P \rightarrow U(d), \beta: P \rightarrow U\left(d^{\prime}\right)$ be h-N-invariant homomorphisms. The inclusions

$$
U\left(c_{\alpha}^{\rho}\right) \times U\left(c_{\beta}^{\rho}\right) \stackrel{\oplus}{\longrightarrow} U\left(c_{\alpha}^{\rho}+c_{\beta}^{\rho}\right)=U\left(c_{\alpha \oplus \beta}^{\rho}\right),
$$

for $\rho \in \operatorname{IrrRep}(P)$, induce an $N$-equivariant inclusion $J_{\alpha} \times J_{\beta} \subseteq J_{\alpha \oplus \beta}$.

Proposition 7.13 The diagram

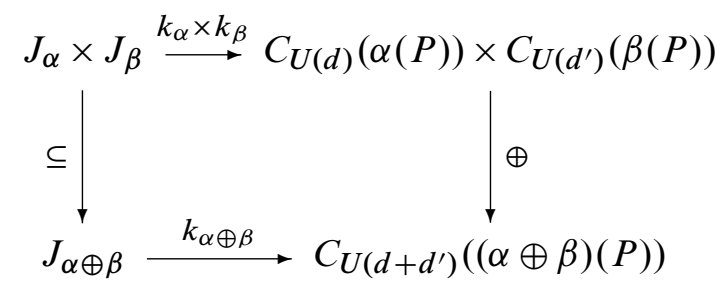

is a commutative diagram in $N^{\mathrm{op}}-\mathbf{R e p}$. 
Proof All homomorphisms in the diagram preserve the right h- $N$-action. For horizontal ones this follows from Proposition 7.10. Let $\mathbb{C}^{d^{\prime}}=\bigoplus_{\rho} W_{\rho}^{\prime}$ be the decomposition such that all irreducible subrepresentations of $W_{\rho}^{\prime}$ are isomorphic to $\rho$; see (7.8). Let $k_{\alpha}^{\rho}: U\left(c_{\alpha}^{\rho}\right) \rightarrow U_{P}\left(W_{\rho}\right), k_{\beta}^{\rho}: U\left(c_{\beta}^{\rho}\right) \rightarrow U_{P}\left(W_{\rho}^{\prime}\right)$ be the isomorphisms defining $k_{\alpha}$ and $k_{\beta}$, respectively. One can choose, for every $\rho \in \operatorname{IrrRep}(P)$, an isomorphism $k_{\alpha \oplus \beta}^{\rho}: U\left(c_{\alpha}^{\rho}+c_{\beta}^{\rho}\right) \rightarrow U_{P}\left(W_{\rho} \oplus W_{\rho}^{\prime}\right)$ such that $\left.k_{\alpha \oplus \beta}^{\rho}\right|_{U\left(c_{\alpha}^{\rho}\right) \times U\left(c_{\beta}^{\rho}\right)}=k_{\alpha}^{\rho} \times k_{\beta}^{\rho}$. For such choices, the commutativity of the diagram follows from definitions.

For an h- $N$-invariant homomorphism $\xi: P \rightarrow U(k)$, define

$$
\begin{aligned}
\mathfrak{J}_{\xi} & :=\left(B J_{\xi}\right)_{p}^{\wedge}, \\
\mathfrak{C}_{\xi} & :=B C_{U(k)}(\xi(P))_{p}^{\wedge}, \\
\mathfrak{M}_{\xi} & :=\left(\operatorname{map}(B P, B U(k))_{B \xi}\right)_{p}^{\wedge} .
\end{aligned}
$$

Proposition 7.17 The diagram

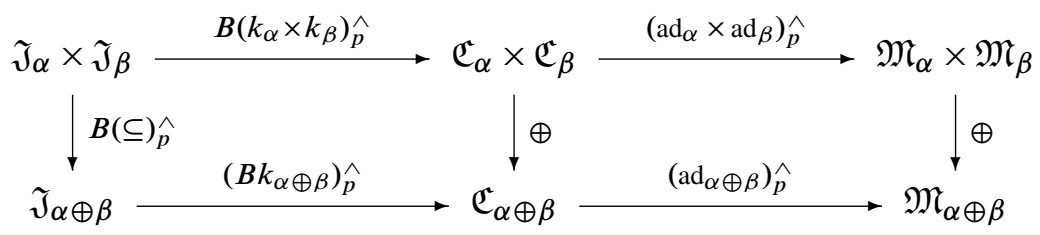

is a commutative diagram in $N^{\mathrm{op}}-\mathbf{H S p}$, and the horizontal maps are $\mathrm{h}-N$-equivalences.

Proof This is a consequence of Proposition 7.5, Proposition 7.10, Proposition 7.13, and the naturality of the ad maps with respect to the target space.

\section{Fibers of equivariant fibrations}

If $p: E \rightarrow B$ is a fibration, then every path $\omega:[0,1] \rightarrow B$ connecting points $b_{0}, b_{1} \in B$ induces a weak homotopy equivalence $\omega_{*}: p^{-1}\left(b_{0}\right) \rightarrow p^{-1}\left(b_{1}\right)$ between the fibers over its endpoints. If $B$ is simply connected, then this map does not depend (up to homotopy) on the choice of a path; in such cases we will write $t_{b_{0}, b_{1}}:=\omega_{*}$. For a commutative diagram

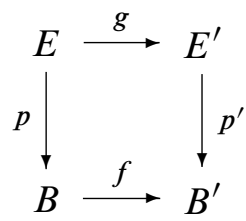


where $p, p^{\prime}$ are fibrations and $B^{\prime}$ is simply connected, we define the induced map between the fibers $F=p^{-1}\left(b_{0}\right)$ and $F^{\prime}=\left(p^{\prime}\right)^{-1}\left(b_{0}^{\prime}\right)$ induced by this diagram as the composition

$$
T_{f}^{g}: F=p^{-1}\left(b_{0}\right) \stackrel{\left.g\right|_{F}}{\longrightarrow}\left(p^{\prime}\right)^{-1}\left(f\left(b_{0}\right)\right) \stackrel{t_{f\left(b_{0}\right), b_{0}^{\prime}}}{\longrightarrow}\left(p^{\prime}\right)^{-1}\left(b_{0}^{\prime}\right)=F^{\prime} .
$$

Clearly it is well-defined up to homotopy.

Let $N$ be a group, $E$ and $B N$-spaces and $p: E \rightarrow B$ an $N$-equivariant fibration over simply connected basis. For every fiber $F=p^{-1}\left(b_{0}\right), b_{0} \in B$, the formula

$$
N \rightarrow[F, F], \quad \eta \mapsto T_{\eta: B \rightarrow B}^{\eta: E \rightarrow E}
$$

defines an h- $N$-action on $F$. If the spaces in the diagram (8.1) are $N$-spaces and the maps are $N$-equivariant, then the induced map between fibers $T_{f}^{g}: F \rightarrow F^{\prime}$ is $\mathrm{h}-N$-equivariant. We will prove that this generalizes, under certain assumptions, to transformations of fibrations which are only h- $N$-equivariant.

Lemma 8.3 Consider a diagram of spaces:

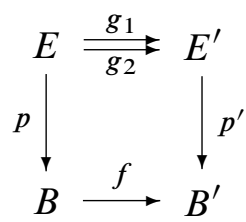

Assume that

- $f p=p^{\prime} g_{1}=p^{\prime} g_{2}=: q$,

- the maps $g_{1}$ and $g_{2}$ are homotopic,

- $\quad p: E \rightarrow B$ and $p^{\prime}: E^{\prime} \rightarrow B^{\prime}$ are simple fibrations with fibers $F=p^{-1}\left(b_{0}\right)$ and $F^{\prime}=\left(p^{\prime}\right)^{-1}\left(f\left(b_{0}\right)\right)$ respectively, where $b_{0} \in B$,

- the homomorphism

$$
\pi_{1}\left(\operatorname{map}\left(E, E^{\prime}\right)_{g_{1}}\right) \stackrel{\operatorname{map}\left(E, p^{\prime}\right)_{*}}{\longrightarrow} \pi_{1}\left(\operatorname{map}\left(E, B^{\prime}\right)_{q}\right)
$$

is surjective.

Then the maps $\left.g_{i}\right|_{F}: F \rightarrow F^{\prime}$ are homotopic for $i=1,2$.

Proof The map $\operatorname{map}\left(E, E^{\prime}\right)_{g_{1}} \rightarrow \operatorname{map}\left(E, B^{\prime}\right)_{q}$ is a fibration with the fiber

$$
M=\left\{g \in \operatorname{map}\left(E, E^{\prime}\right)_{g_{1}}: p^{\prime} g=q\right\} .
$$


By the last assumption $M$ is path-connected. Furthermore, the image of the restriction $M \rightarrow \operatorname{map}\left(F, E^{\prime}\right)$ is contained in $\operatorname{map}\left(F, F^{\prime}\right)$. Hence, $g_{1}, g_{2} \in M$ restrict to homotopic maps $\left.g_{1}\right|_{F},\left.g_{2}\right|_{F} \in \operatorname{map}\left(F, F^{\prime}\right)$.

Proposition 8.4 Consider a diagram of simply connected $N$-spaces

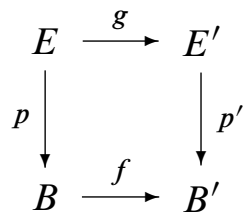

that is commutative as a diagram of spaces. Assume that the maps $p$ and $p^{\prime}$ are $N-$ equivariant fibrations, and the maps $f$ and $g$ are h- $N$-equivariant. Let $F=p^{-1}\left(b_{0}\right)$ and $F^{\prime}=\left(p^{\prime}\right)^{-1}\left(b_{0}^{\prime}\right)$ be the fibers of $p$ and $p^{\prime}$ respectively; assume that they are also simply connected. If the homomorphism

$$
p_{*}^{\prime}: \pi_{1} \operatorname{map}\left(E, E^{\prime}\right)_{g} \rightarrow \pi_{1} \operatorname{map}\left(E, B^{\prime}\right)_{p^{\prime} g}
$$

is surjective, then the induced map $T_{f}^{g}: F \rightarrow F^{\prime}$ between the fibers is h- $N$-equivariant.

Proof Fix $\eta \in N$. Denote

$$
\begin{array}{ll}
f_{1}=B \stackrel{\eta}{\longrightarrow} B \stackrel{f}{\longrightarrow} B^{\prime}, & g_{1}=E \stackrel{\eta}{\longrightarrow} E \stackrel{g}{\longrightarrow} E^{\prime}, \\
f_{2}=B \stackrel{f}{\longrightarrow} B^{\prime} \stackrel{\eta}{\longrightarrow} B^{\prime}, & g_{2}=E \stackrel{g}{\longrightarrow} E^{\prime} \stackrel{\eta}{\longrightarrow} E^{\prime} .
\end{array}
$$

We have to prove that the commutative diagrams

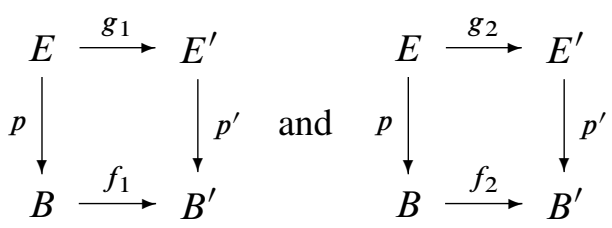

induce homotopic maps between the fibers, ie that the maps $T_{f_{1}}^{g_{1}}$ and $T_{f_{2}}^{g_{2}}$ are homotopic. Let $F: B \times I \rightarrow B^{\prime}$ be a homotopy between $f_{2}=\left.F\right|_{B \times 0}$ and $f_{1}=\left.F\right|_{B \times 1}$, and let $L: E \times I \rightarrow E^{\prime}$ be a lifting extension fitting into the diagram:

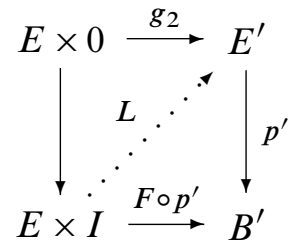


Denote $g_{2}^{\prime}=\left.L\right|_{E \times 1}: E \rightarrow E^{\prime}$. The maps $T_{f_{2}}^{g_{2}}$ and $T_{f_{1}}^{g_{2}^{\prime}}$ are homotopic since $(F, L)$ is a homotopy between the transformations $\left(f_{2}, g_{2}\right)$ and $\left(f_{1}, g_{2}^{\prime}\right)$. Finally, the diagram

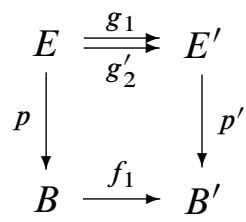

satisfies the assumptions of Lemma 8.3 since the homomorphism

$$
\begin{aligned}
\pi_{1}\left(\operatorname{map}\left(E, E^{\prime}\right)_{g_{1}}\right) & =\pi_{1}\left(\operatorname{map}\left(E, E^{\prime}\right)_{g \eta}\right) \\
& \stackrel{\operatorname{map}\left(\eta^{-1}, E^{\prime}\right)_{*}}{\longrightarrow} \pi_{1}\left(\operatorname{map}\left(E, E^{\prime}\right)_{g}\right) \\
& \stackrel{\operatorname{map}\left(E, p^{\prime}\right)_{*}}{\longrightarrow} \pi_{1}\left(\operatorname{map}\left(E, B^{\prime}\right)_{p^{\prime} g}\right) \\
& \stackrel{\operatorname{map}\left(\eta, B^{\prime}\right)_{*}}{\longrightarrow} \pi_{1}\left(\operatorname{map}\left(E, B^{\prime}\right)_{p^{\prime} g \eta}\right) \\
& =\pi_{1}\left(\operatorname{map}\left(E, B^{\prime}\right)_{p^{\prime} g_{1}}\right)
\end{aligned}
$$

is an epimorphism. Thus the maps $\left.g_{1}\right|_{F},\left.g_{2}^{\prime}\right|_{F}: F \rightarrow\left(p^{\prime}\right)^{-1}\left(f_{1}\left(b_{0}\right)\right)$ and are homotopic. Finally we obtain the sequence of homotopic maps $F \rightarrow F^{\prime}$

$$
T_{f_{1}}^{g_{1}}=\left.\left.t_{f_{1}\left(b_{0}\right), b_{0}^{\prime}} \circ g_{1}\right|_{F} \sim t_{f_{1}\left(b_{0}\right), b_{0}^{\prime}} \circ g_{2}^{\prime}\right|_{F}=T_{f_{1}}^{g_{2}^{\prime}} \sim T_{f_{2}}^{g_{2}},
$$

which ends the proof.

\section{Equivalence of homotopy centralizers}

Proposition 8.5 Let $G$ and $H \subseteq H^{\prime}$ be compact Lie groups, and let $\alpha$ : $G \rightarrow H$ be a homomorphism. Assume that for every $p$-stubborn subgroup $P \subseteq G$, the centralizers $C_{H}(\alpha(P))$ and $C_{H^{\prime}}(\alpha(P))$ are equal. Then the map

$$
\operatorname{map}\left(B G_{p}^{\wedge}, B H_{p}^{\wedge}\right)_{B \alpha} \rightarrow \operatorname{map}\left(B G_{p}^{\wedge},\left(B H^{\prime}\right)_{p}^{\wedge}\right)_{B \alpha}
$$

is a homotopy equivalence.

Proof Let $i: H \rightarrow H^{\prime}$ be the inclusion. Denote $\operatorname{map}\left(B G_{p}^{\wedge}, B H_{p}^{\wedge}\right)_{(\alpha)}:=\left\{f: B G_{p}^{\wedge} \rightarrow B H_{p}^{\wedge}\right.$ : for all $\left.G / P \in \mathcal{R}_{p}(G),\left.f\right|_{B P_{p}^{\wedge}} \sim\left(\left.B \alpha\right|_{P}\right)_{p}^{\wedge}\right\}$ 
There is a commutative diagram

$$
\begin{aligned}
& \operatorname{map}\left(B G, B H_{p}^{\wedge}\right)_{(\alpha)} \longrightarrow \operatorname{i*}\left(B G,\left(B H^{\prime}\right)_{p}^{\wedge}\right)_{(i \circ \alpha)} \\
& \simeq \text { Theorem } 2.11 \quad \simeq \downarrow \text { Theorem } 2.11
\end{aligned}
$$

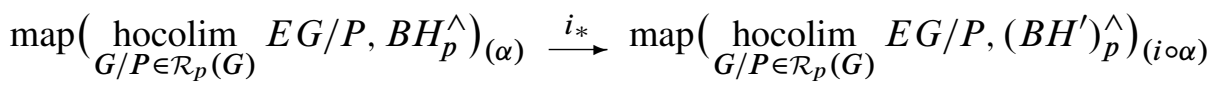

$$
\begin{aligned}
& \simeq \downarrow \quad \simeq \\
& \operatorname{holim}_{G / P \in \mathcal{R}_{p}(G)} \operatorname{map}\left(E G / P, B H_{p}^{\wedge}\right)_{\left.\alpha\right|_{P}} \stackrel{i_{*}}{\longrightarrow} \underset{G / P \in \mathcal{R}_{p}(G)}{\operatorname{holim}} \operatorname{map}\left(E G / P,\left(B H^{\prime}\right)_{p}^{\wedge}\right)_{\left.i \circ \alpha\right|_{P}}
\end{aligned}
$$

with horizontal arrows induced by $i: H \rightarrow H^{\prime}$, and vertical ones being homotopy equivalences. Since the bottom horizontal map is an equivalence by assumptions, so is the upper one. By restricting to the component of $(B \alpha)_{p}^{\wedge}$ on the left-hand side and that of $(B i \circ \alpha)_{p}^{\wedge}$ on the right-hand side, we obtain the conclusion.

Proposition 8.6 Let $k, l$ be positive integers. If $P \subseteq U(k), Q \subseteq U(l)$ are $p$-stubborn subgroups, then

$$
C_{U(k+l)}(P \times Q)=C_{U(k) \times U(l)}(P \times Q)=C_{U(k)}(P) \times C_{U(l)}(Q) .
$$

Proof Let $\iota_{P}: P \subseteq U(k), \iota_{Q}: Q \subseteq U(l)$ denote the inclusions and $\pi_{P}: P \times Q \rightarrow P$, $\pi_{Q}: P \times Q \rightarrow Q$ the projections. The representation $\iota_{P}$ does not contain a trivial summand; otherwise the normalizer of $P$ in $U(k)$ would have a greater dimension than $P$ which contradicts $p$-stubbornness. Every irreducible representation of $P \times Q$ has the form $\rho \bar{\otimes} \sigma:=\left(\rho \circ \pi_{P}\right) \otimes\left(\sigma \circ \pi_{Q}\right)$, where $\rho$ and $\sigma$ are irreducible representations of $P$ and $Q$ respectively. If $\iota_{P} \circ \pi_{P}$ contains a subrepresentation isomorphic to $\rho \bar{\otimes} \sigma$, then $\sigma$ is trivial and $\rho$ is nontrivial; if it is contained in $\iota_{Q} \circ \pi_{Q}$ then $\rho$ is nontrivial. As a consequence, there exists no irreducible representation of $P \times Q$ which is contained as a summand in both $\iota_{P} \circ \pi_{P}$ and $\iota_{Q} \circ \pi_{Q}$. By Schur's lemma, we obtain

$$
\begin{aligned}
C_{U(k+l)}(P \times Q) & \cong C_{U(k+l)}\left(\left(\iota_{P} \circ \pi_{P}\right) \oplus\left(\iota_{Q} \circ \pi_{Q}\right)\right) \\
& \cong C_{U(k)}\left(\iota_{P}\right) \times C_{U(l)}\left(\iota_{Q}\right) \cong C_{U(k)}(P) \times C_{U(l)}(Q) .
\end{aligned}
$$

Proposition 8.7 Let $N$ be a finite group and $P$ a $p$-toral h- $N$-group. Assume that $\alpha: P \rightarrow U(d)$ and $\beta: P \rightarrow U\left(d^{\prime}\right)$ are h- $N$-invariant homomorphisms. Then the map

$$
\operatorname{map}\left(\mathfrak{J}_{\alpha} \times \mathfrak{J}_{\beta}, \mathfrak{J}_{\alpha} \times \mathfrak{J}_{\beta}\right)_{\text {id }} \rightarrow \operatorname{map}\left(\mathfrak{J}_{\alpha} \times \mathfrak{J}_{\beta}, \mathfrak{J}_{\alpha \oplus \beta}\right)_{B(\subseteq) \hat{p}},
$$

induced by the inclusion $J_{\alpha} \times J_{\beta} \subseteq J_{\alpha \oplus \beta}$, is a homotopy equivalence. 
Proof Let $Q$ be a $p$-stubborn subgroup of $J_{\alpha} \times J_{\beta}$. By [5, Theorem 1.6], every $p$-stubborn subgroup of $J_{\alpha} \times J_{\beta}$ has the form

$$
\prod Q_{\rho} \times \prod Q_{\rho}^{\prime} \subseteq \prod U\left(c_{\alpha}^{\rho}\right) \times \prod U\left(c_{\beta}^{\rho}\right),
$$

where the products are indexed over $\rho \in \operatorname{IrrRep}(P)$, and $Q_{\rho} \subseteq U\left(c_{\alpha}^{\rho}\right), Q_{\rho}^{\prime} \subseteq U\left(c_{\beta}^{\rho}\right)$ are $p$-stubborn subgroups. Then

$$
\begin{aligned}
C_{J_{\alpha}(P) \times J_{\beta}(P)}(Q) & =\prod C_{U\left(c_{\alpha}^{\rho}\right)}\left(Q_{\rho}\right) \times \prod C_{U\left(c_{\beta}^{\rho}\right)}\left(Q_{\rho}^{\prime}\right) \\
& \cong \prod C_{U\left(c_{\alpha}^{\rho}\right) \times U\left(c_{\beta}^{\rho}\right)}\left(Q_{\rho} \times Q_{\rho}^{\prime}\right) \\
& \cong \prod C_{U\left(c_{\alpha}^{\rho}+c_{\beta}^{\rho}\right)}\left(Q_{\rho} \times Q_{\rho}^{\prime}\right)=C_{J_{\alpha+\beta}(P)}(Q),
\end{aligned}
$$

where again the products are indexed over $\rho \in \operatorname{IrrRep}(P)$, and the last isomorphism follows from Proposition 8.6. The conclusion follows from Proposition 8.5.

\section{Equivariant equivalence of fibers}

Finally, we return to the situation of Theorem 4.9. Let $G$ be a compact connected Lie group, $P \subseteq G$ a $p$-stubborn subgroup, and $\mu, v \mathcal{P}$-characters of $G$ with dimensions $d, d^{\prime}$, respectively. Denote $N=N_{G}(P) / P, \alpha:=\mu_{P}: P \rightarrow U(d), \beta:=$ $v_{P}: P \rightarrow U\left(d^{\prime}\right)$. As before, $\alpha$ and $\beta$ are h- $N$-invariant. Let

$$
\mathfrak{M}_{\alpha}^{G} \tilde{\times} \mathfrak{M}_{\beta}^{G}:=\operatorname{map}\left(E G \times_{G} G / P,\left(E U\left(d+d^{\prime}\right) /\left(U(d) \times U\left(d^{\prime}\right)\right)\right)_{p}^{\wedge}\right)_{B(\alpha \oplus \beta)_{p}^{\wedge}}
$$

and, for an arbitrary h- $N$-invariant homomorphism $\xi: P \rightarrow U(k)$, let

$$
\mathfrak{M}_{\xi}^{G}:=\operatorname{map}\left(E G \times_{G} G / P, B U(k)_{p}^{\wedge}\right)_{B \xi} .
$$

The inclusion $U(d) \times U\left(d^{\prime}\right) \subseteq U\left(d+d^{\prime}\right)$ induces an $N$-equivariant fibration

$$
m_{\alpha, \beta}^{G}: \mathfrak{M}_{\alpha}^{G} \tilde{\times} \mathfrak{M}_{\beta}^{G} \rightarrow \mathfrak{M}_{\alpha \oplus \beta}^{G},
$$

where the right action of $N$ comes from the left action on $E G \times_{G} G / P$.

Proposition 8.8 The homotopy fiber of $m_{\alpha, \beta}^{G}$ is h-N-equivalent to $\operatorname{Fib}_{\mu, \nu}^{p}(G / P)$.

Proof Homotopy equivalence of these spaces comes from the definition of the space $\operatorname{Fib}_{\mu, \nu}^{p}(G / P)$; see (3.2), (4.3). Also the definitions of the homotopy $N$-action on $\operatorname{Fib}_{\mu, \nu}^{p}(G / P)$ (3.3) and on the homotopy fiber of $m_{\alpha, \beta}^{G}$ (8.2) coincide.

Recall (7.12) that the inclusion $J_{\alpha} \times J_{\beta} \subseteq J_{\alpha \oplus \beta}$ is $N$-equivariant. Define an $N$-space

$$
\mathfrak{J}_{\alpha} \tilde{\times} \mathfrak{J}_{\beta}:=\left(E J_{\alpha \oplus \beta} /\left(J_{\alpha} \times J_{\beta}\right)\right)_{p}^{\wedge}
$$


and an $N$-equivariant fibration

$$
j_{\alpha, \beta}: \mathfrak{J}_{\alpha} \tilde{\times} \mathfrak{J}_{\beta}=\left(E J_{\alpha \oplus \beta} /\left(J_{\alpha} \times J_{\beta}\right)\right)_{p}^{\wedge} \rightarrow\left(E J_{\alpha \oplus \beta} / J_{\alpha \oplus \beta}\right)_{p}^{\wedge}=\mathfrak{J}_{\alpha \oplus \beta} .
$$

Clearly the space $\mathfrak{G}_{\alpha, \beta}(4.7)$ is homotopy $N$-equivalent to the fiber of $j_{\alpha, \beta}$.

Proof of Theorem 4.9 There is a commutative diagram in $N^{\mathrm{op}}-\mathbf{H S p}$

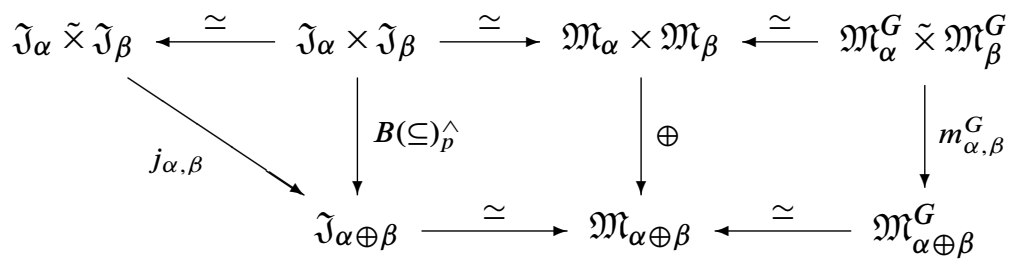

where the middle square is the diagram Proposition 7.17, and the remaining equivalences are induced by h- $N$-equivalences $E G \times_{G} G / P \simeq B P$ and $E J_{\alpha \oplus \beta} \simeq E J_{\alpha} \times E J_{\beta}$. Since $m_{\alpha, \beta}^{G}$ is a fibration, there exists a strictly commutative diagram

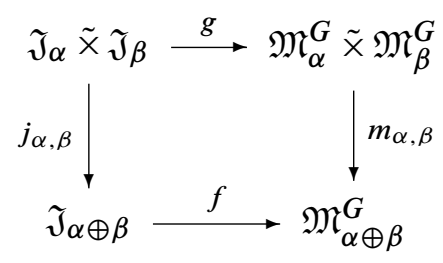

such that the horizontal maps $f$ and $g$ are h- $N$-equivalences. Let $T_{f}^{g}: \mathfrak{G}_{\alpha, \beta} \rightarrow$ $\operatorname{Fib}_{\mu, \nu}^{p}(G / P)$ be the induced map between the homotopy fibers of vertical fibrations. By Proposition 8.7, this diagram satisfies the assumptions of Proposition 8.4. Then the map $T_{f}^{g}$ is an h- $N$-equivariant map and a homotopy equivalence (since $f$ and $g$ are homotopy equivalences).

\section{References}

[1] J L Alperin, P Fong, Weights for symmetric and general linear groups, J. Algebra 131 (1990) 2-22 MR1054996

[2] M Aschbacher, R Kessar, B Oliver, Fusion systems in algebra and topology, London Mathematical Society Lecture Note Series 391, Cambridge Univ. Press, MA (2011) MR2848834

[3] A K Bousfield, D M Kan, Homotopy limits, completions and localizations, Lecture Notes in Mathematics 304, Springer, Berlin (1972) MR0365573 
[4] W Dwyer, A Zabrodsky, Maps between classifying spaces, from: "Algebraic topology”, (J Aguadé, R Kane, editors), Lecture Notes in Math. 1298, Springer, Berlin (1987) 106-119 MR928826

[5] S Jackowski, J McClure, B Oliver, Homotopy classification of self-maps of BG via G-actions I, Ann. of Math. 135 (1992) 183-226 MR1147962

[6] S Jackowski, J McClure, B Oliver, Homotopy classification of self-maps of BG via G-actions II, Ann. of Math. 135 (1992) 227-270 MR1154593

[7] S Jackowski, J McClure, B Oliver, Maps between classifying spaces revisited, from: “The Čech centennial”, (M Cenkl, H Miller, editors), Contemp. Math. 181, Amer. Math. Soc., Providence, RI (1995) 263-298 MR1320996

[8] S Jackowski, B Oliver, Vector bundles over classifying spaces of compact Lie groups, Acta Math. 176 (1996) 109-143 MR1395671

[9] W Lubawski, K Ziemiański, Low dimensional homotopy representations of unitary groups In preparation

[10] D Notbohm, Maps between classifying spaces, Math. Z. 207 (1991) 153-168 MR1106820

[11] B Oliver, Higher limits via Steinberg representations, Comm. Algebra 22 (1994) 13811393 MR1261265

[12] B Oliver, p-stubborn subgroups of classical compact Lie groups, J. Pure Appl. Algebra 92 (1994) 55-78 MR1259669

[13] D Sullivan, Geometric topology: localization, periodicity, and Galois symmetry, Mimeographed notes, Massachusetts Institute of Technology (1970) IATEX version available at http://www.maths.ed.ac.uk/ aar/books/gtop.pdf

[14] Z Wojtkowiak, On maps from ho $\lim _{\longrightarrow} F$ to $\mathbf{Z}$, from: "Algebraic topology", (J Aguadé, R Kane, editors), Lecture Notes in Math. 1298, Springer, Berlin (1987) 227-236 MR928836

Institute of Mathematics, Polish Academy of Sciences

Śniadeckich 8, 00-956 Warszawa, Poland

Faculty of Mathematics, Informatics and Mechanics, University of Warsaw

Banacha 2, 02-097, 02-097 Warszawa, Poland

w.lubawski@gmail.com, ziemians@mimuw.edu.pl

Received: 26 June 2014 Revised: 26 October 2015 
